\title{
Relationship between X-Ray Emission and Absorption Spectroscopy and the Local H-bond Environment in Water
}

\author{
Iurii Zhovtobriukh ${ }^{a}$, Nicholas A. Besley ${ }^{b}$, Thomas Fransson ${ }^{c^{\perp}}$, Anders \\ Nilsson $^{a}$, and Lars G.M. Pettersson ${ }^{* a}$ \\ ${ }^{a}$ FYSIKUM, Stockholm University, Albanova University Center, \\ SE-106 91 Stockholm, Sweden. \\ ${ }^{b}$ School of Chemistry, The University of Nottingham, University Park, \\ Nottingham NG72RD, United Kingdom \\ ${ }^{c}$ Stanford PULSE Institute, SLAC National Accelerator Laboratory, Menlo \\ Park, California 94025, USA. \\ ${ }^{\perp}$ Present address: Interdisciplinary Center for Scientific \\ Computing, University of Heidelberg, Im Neuenheimer Feld 205, \\ 69120 Heidelberg, Germany
}

*E-mail:lgm@fysik.su.se;Tel: +46(8)55378712

\begin{abstract}
The connection between specific spectrum features in the water X-ray absorption (XAS) and X-ray emission (XES) spectra and the local H-bond coordination is studied based on structures obtained from path-integral molecular dynamics simulations using either the opt-PBE-vdW density functional or the MB-pol force field. Computing the XES spectrum using all molecules in a snapshot results in only one peak in the lone-pair $\left(1 b_{1}\right)$ region while the experiment shows two peaks separated by 0.8 $0.9 \mathrm{eV}$. Different H-bond configurations were classified based on the local structure index (LSI) and
\end{abstract}


a geometrical H-bond cone criterion. We find that tetrahedrally coordinated molecules characterized by high LSI values and two strong donated and two strong accepted H-bonds contribute to the low energy $1 b_{1}$ emission peak and to the post-edge region in absorption. Molecules with asymmetric $\mathrm{H}$ bond environment with one strong accepted and one strong donated H-bond and low LSI values give rise to the high energy $1 b_{1}$ peak in the emission spectrum and mainly contribute to the pre-edge and main-edge in the absorption spectrum. The $1 b_{1}$ peak splitting can be increased to $0.62 \mathrm{eV}$ by imposing constraints on the H-bond length, i.e. for very tetrahedral structures short H-bonds (less than $2.68 \AA$ ) and for very asymmetric structures elongated H-bonds (longer than $2.8 \AA$ ). Such structures are present, but underrepresented, in the simulations which give more of an average of the two extremes. 


\section{INTRODUCTION}

Water is one of the most common and important compounds on our planet and is vitally important in industry where it finds use, e.g., as solvent, diluting compound, efficient heat transport and cooling agent. Water participates in most chemical reactions in biological systems as an essential compound or as the chemical environment in which these reactions occur. It is also the most anomalous liquid ${ }^{1}$ with a great number of unusual properties, such as increasing density upon melting, density maximum at $4^{\circ} \mathrm{C}$, decreasing viscosity under pressure, high surface tension and many more ${ }^{2}$. The structural anomalies are enhanced in the supercooled region, but they are also present at ambient conditions where the relevant physical and biochemical processes occur. Obviously, the origin of these macroscopic anomalies should be connected with structural and dynamical properties of the hydrogen-bond (H-bond) network in water ${ }^{3,4}$. Here we will combine different X-ray spectroscopy data to show that this network at ambient conditions is still not well described in theoretical models, in spite of significant progress in molecular dynamics (MD) simulations of water ${ }^{5}$

The common picture of the structure of the H-bond network in water has been as a predominantly tetrahedral $\mathrm{H}$-bond network with around four $\mathrm{H}$-bonds per molecule, similar to ordinary ice, but more distorted due to thermal motion. In 2004 the publication by Wernet et al. ${ }^{6}$ started a new round of debate around water structure at ambient conditions by proposing an alternative picture of the microscopic water structure where the majority of water molecules has on average only two well-defined H-bonds (one donated and one accepted), i.e. asymmetrically distorted local configurations. This proposal was based on X-ray absorption spectroscopy (XAS) and X-ray Raman scattering (XRS) coupled with theoretical spectrum simulations. The 
interpretation was challenged by Saykally and coworkers ${ }^{7}$, however based on data that suffered from saturation effects ${ }^{8}$. Later, using also X-ray emission spectroscopy $(\mathrm{XES})^{9-11}$, where the liquid exhibits two sharp peaks with temperature-dependent ratio in the lone-pair region, and small-angle X-ray scattering (SAXS) data ${ }^{9,12,13}$ a new picture of ambient water was proposed based on fluctuations between two dominating local structural states in the liquid ${ }^{3,14,15}$ : low density water (LDL) with tetrahedral local coordination and high density water (HDL) with asymmetrically distorted first hydration shell and broken/weakened H-bonds. This hypothesis is related to the firstorder liquid-liquid transition that was originally proposed by Poole et al. ${ }^{16}$ to explain water anomalies in the deeply supercooled regime and which has been examined extensively ${ }^{17-28}$.

The interpretation of the SAXS data, as indicating fluctuating density heterogeneities, was challenged $^{29-32}$, in part based on a data set covering a smaller $Q$-range and on simulations that did not reproduce the enhancement at low $Q^{29}$ upon supercooling. However, the picture of structural fluctuations leading to local density fluctuations was supported by Overduin and Patey ${ }^{33}$ based on analysis of computed SAXS from simulations of TIP4P $/ 2005^{34}$ water. Measurements of SAXS at deep supercooling ${ }^{12,35}$, where the deviation from normal liquid behavior becomes very strong, further underline this picture of structural fluctuations.

A two-state picture of water leading to predominance at ambient conditions of close-packing of molecules (HDL, favored by entropy), but with local fluctuations into tetrahedral environments (LDL, favored by enthalpy) is fully consistent with thermodynamics, where such models have been shown to quantitatively reproduce the thermodynamic properties of water over a wide range of temperatures and pressures ${ }^{21,24,36,37}$. Polarization-dependent vibrational Raman spectroscopy at the $\mathrm{OH}$-stretch in $\mathrm{H}_{2} \mathrm{O}$ has concluded a 3:1 ratio at ambient conditions between molecules in disordered versus symmetric tetrahedral local environment ${ }^{38}$; this is consistent with XAS and $\mathrm{XES}^{6,9-11}$. Maréchal ${ }^{39}$ has shown that the full experimental IR spectrum $\left(0<\tilde{v}<4000 \mathrm{~cm}^{-1}\right)$ of 
liquid water can be decomposed in terms of a temperature-dependent $\left(-4^{\circ} \mathrm{C}\right.$ to $\left.80^{\circ} \mathrm{C}\right)$ combination of two complete, temperature-independent spectra called ground state (i.e. LDL) and excited (i.e. HDL); this is consistent with the fluctuating mixture in the ambient regime ${ }^{3,15}$. It should be noted that, in contrast to decomposition of the rather featureless $\mathrm{OH}$-stretch peak, which many authors have done using two or more Gaussians, a decomposition of the entire spectral region $(0<\tilde{v}<$ $4000 \mathrm{~cm}^{-1}$ ) in terms of two complete fixed spectra represents a very strong constraint. In addition, since the decomposition of the full spectrum can be done in terms of two full temperatureindependent spectra, the arguments raised by Geissler and coworkers ${ }^{40,41}$ against interpreting isosbestic points as due to interconversion between species do not apply in this case.

It is here important to distinguish between $\mathrm{H}_{2} \mathrm{O}$ and $\mathrm{HDO}$ vibrational data since the line shapes are dramatically different. Geissler and co-workers have made the case to describe the HDO vibrational spectra as due to a continuous distribution of structures that results in the broad and almost featureless spectrum. However, the $\mathrm{H}_{2} \mathrm{O}$ spectrum shows three spectral features that vary in intensity with only small shifts as the temperature changes and we argue that this cannot be described based on a continuous homogeneous distribution of structures. This will become more apparent in the comparison below of the $\mathrm{H}_{2} \mathrm{O} \mathrm{OH}$ stretch spectra between $\mathrm{MB}^{-p l^{42}}$ water and experiment.

In a recent study, the transition between pure HDL and LDL phases in the ultraviscous regime was followed experimentally using wide-angle X-ray scattering to monitor the density and X-ray photon correlation spectroscopy to measure the diffusive dynamics ${ }^{22}$. Simulations finding two freeenergy basins for the liquid in the deeply supercooled regime at elevated pressure ${ }^{20,43,44}$ have been strongly debated ${ }^{45-52}$, but are consistent with this result. Furthermore, the claim that the liquidliquid transition in simulations of the ST2 water model ${ }^{53}$ in reality is a liquid-solid transition ${ }^{47,48}$ has been shown to be faulty and due to an error in the implementation of the hybrid Monte Carlo 
sampling protocol ${ }^{54}$. Very recently, SAXS measurements of the isothermal compressibility and correlation length for $\mathrm{H}_{2} \mathrm{O}$ and $\mathrm{D}_{2} \mathrm{O}$ were reported down to $227 \mathrm{~K}^{35}$. Both properties were found to exhibit a maximum fully in agreement with a Widom line emanating from a critical point terminating a liquid-liquid coexistence line $\mathrm{e}^{35}$. There is thus mounting evidence for a two-state picture of water leading to fluctuations in the ambient regime. Recent reviews summarize the present picture ${ }^{3,4}$.

The interpretation of the XAS spectroscopic features in terms of various H-bond configurations is well-established, although the magnitude of distortions is under debate ${ }^{6-8,55-64}$. In the water molecule the lowest unoccupied molecular orbitals have large spatial extent and they are polarized towards the hydrogen atoms. Due to this, their shape and orbital energies are sensitive to formation of donated H-bonds making XAS a very sensitive tool to probe the donated H-bond local structure. In addition, the time scale of the core excitation process is around attosecond, while the typical timescale of hydrogen bond dynamics is around hundreds of femtoseconds ${ }^{65}$ which allows the instantaneous water structure to be probed.

The XAS water spectrum is usually divided into three main parts: sharp pre-edge centered at $535 \mathrm{eV}$, strong main-edge centered around $537 \mathrm{eV}$ and strong and extended post-edge centered at $541 \mathrm{eV}$. The pre-edge is associated with weakened and broken H-bonds ${ }^{6,59,66-69}$, while the postedge feature is due to intact $\mathrm{H}$-bonds with the main contribution from molecules with fully tetrahedrally coordinated H-bonds ${ }^{66-69}$. The main-edge is also sensitive to H-bond distortions, but additionally linked up with collapse of the second hydration shell ${ }^{14,60,70}$. Many attempts have been made to simulate the water XAS spectrum using different theoretical approaches, such as transition potential density functional theory $(\mathrm{TPDFT})^{57,71-74}$, the Bethe-Salpeter equation in different approximations $^{75,76}$, time-dependent density functional theory (TDDFT) ${ }^{77}$, and excited-core-hole $(\mathrm{XCH})^{78}$. These approaches generate the correct assignment for the general XAS spectrum 
features, but none of them can fully reproduce the XAS water spectrum in all spectrum regions (pre-, main- and post-edge $)^{79,80}$.

X-ray emission spectroscopy probes the occupied states via electronic radiative transitions from occupied levels to the core hole, making it complementary to $\mathrm{XAS}^{9}$. For non-resonant excitation, the XES spectrum of gas phase water exhibits three main features which arise due to electron transitions from the valence bonding $1 b_{2}, 3 a_{1}$ and non-bonding $1 b_{1}$ molecular orbitals, where the $1 b_{1}$ emission peak is sharp while the $1 b_{2}$ and $3 a_{1}$ peaks are smeared due to vibrational excitations ${ }^{10,}$ 11, 81 . In contrast to the gas phase, the XES spectrum of liquid water shows a split of the lone-pair $1 b_{1}$ peak in two ${ }^{10,11,82-86}$ which are usually labelled as $1 b_{1}^{\prime}$ (low emission energy) and $1 b_{1}^{\prime \prime}$ (high emission energy). A similar split of the $1 b_{1}$ peak is also observed in the XES spectrum of ice measured in vacuum and at low temperature, but it is known that the X-ray beam in this case can induce a pressure wave upon excitation, transforming the region around the absorbing atom into high density amorphous ice and thereby generating two peaks. In liquid water there is an equilibrium with fluctuations where such a transformation would be converted back to the ground state on a picosecond timescale ${ }^{70,87}$. In the case of crystalline ice measured at $-10{ }^{\circ} \mathrm{C}$, i.e. with fast equilibration with the gas phase, the spectrum is dominated by the peak at low emission energy ${ }^{88}$.

Several explanations have been proposed to account for this split. Fuchs et al. ${ }^{82,89}$ proposed that the $1 b_{1}^{\prime}$ peak (low emission energy) comes from $\mathrm{OH}^{-}$species obtained from water molecules due to ultrafast dissociation, which is promoted by the presence of the intermolecular H-bonds, while the $1 b_{1}^{\prime \prime}$ peak (high emission energy) was attributed to intact water molecules. This conclusion was made based on comparison between non-resonantly excited emission spectra of $\mathrm{H}_{2} \mathrm{O}$ and $\mathrm{D}_{2} \mathrm{O}$ and resonantly excited emission spectra of $\mathrm{NaOH}$ and $\mathrm{NaOD}$ water solutions. A very recent vibrationally resolved study ${ }^{90}$, however, shows that dissociation indeed contributes to the $1 b_{1}^{\prime}$ peak, but only for pre-edge excitation; above the ionization limit dissociation contributes 
insignificantly with some intensity between the $1 b_{1}^{\prime}$ and $1 b_{1}^{\prime \prime}$ peaks. Odelius ${ }^{91,92}$ assigned the two peaks as $3 a_{1}$ bonding and $1 b_{1}$ lone-pair based on simulated spectra, but measurement of the symmetry of the two peaks resulted in both being of $1 b_{1}$ symmetry ${ }^{93}, 94$. An alternative interpretation instead explains the split based on the two structural motifs HDL and LDL ${ }^{3,8-11,14,}$ 30, 93, 95-99. This interpretation is supported by measurements of the symmetry of the two peaks ${ }^{93,94}$ and is furthermore consistent with X-ray photoelectron spectroscopy (XPS) where both the oxygen $1 s$ and the $1 b_{1}$ valence photoelectron peaks are broad enough to support two unresolved peaks ${ }^{100-}$ ${ }^{102}$. On the other hand, the creation of the oxygen $1 s$ core-hole generates dynamics involving the hydrogen atoms ${ }^{95,96,103-105}$. The oxygen $1 s$ core-hole lifetime is about $4 \mathrm{fs}^{106}$, which means that dynamical effects are important in the XE process ${ }^{82,} 89,91,92,95,96,103-105,107$. However, the dominating effect of the dynamics is a redistribution of intensity without the appearance of new features, as demonstrated in model systems of water ${ }^{95,96}$ and recently by spectrum simulations of liquid alcohols ${ }^{108,109}$ and water ${ }^{110}$.

All previous attempts to simulate the XES spectrum of water ${ }^{10,11,91,92,110}$ have usually been performed without connection to the XAS spectrum simulation. The converse is also true in case of XAS ${ }^{71,75-78,111}$. The final state of XAS is the initial state in XES and both spectra contain features which can be attributed to the different H-bond situations. This correlation between XAS and XES spectroscopy and H-bond coordination has furthermore been demonstrated experimentally $9,96,112$. A structural model of water should thus consider and be consistent with both XAS and XES and ideally reproduce all other available experimental data. Here we combine XAS and non-resonant XES calculations based on the same structures to determine constraints on local structures that can simultaneously reproduce XAS and XES. The first goal of this article is to correlate different spectrum features in XES and XAS with the local H-bond coordination. The second goal is to show that the split in the $1 b_{1}$ emission peak can be reproduced if one assumes two sets of constrained 
structures - very tetrahedrally coordinated structures with four strong and short H-bonds and highly asymmetrical structures with one donated and one accepted H-bond. Such structures are present in simulated water, but only as a minor fraction where the simulations seem rather to give an average of the two extremes seen in real water.

\section{COMPUTATIONAL DETAILS}

When different H-bond coordinated structures are considered, some order parameter is needed to distinguish the different structures. Here we will use the local structure index (LSI) of Sasaki and Sasai ${ }^{113}$ together with the X-ray spectroscopy based H-bond definition of Wernet et al. ${ }^{6}$

The definition of the LSI is as follows. If the distances between the central water molecule and its nearest neighbors are ordered such that $r_{1}<r_{2}<\ldots<r_{i}<r_{i+1}<\ldots<r_{n}<3.7 \AA<r_{n+1}$, the LSI is defined as:

$$
L S I=\frac{1}{n} \sum_{i=1}^{n}(\Delta(i)-\bar{\Delta})^{2}
$$

where $\Delta(i)=r_{i+1}-r_{i}$ and $\bar{\Delta}$ is the arithmetic mean of $\Delta(i)$. The LSI measures the degree of disorder out to the second hydration shell where, in case of mainly tetrahedrally coordinated water molecules, the difference $\Delta(i)-\bar{\Delta}$ demonstrates large jumps due to the molecules lying in wellseparated first and second hydration shells which results in high LSI values. On the other hand, for molecules with distorted and asymmetrical environment, the differences $\Delta(i)-\bar{\Delta}$ will be small due to the presence of interstitial molecules between the first and second hydration shells. The LSI parameter has been shown ${ }^{114} 115$ to exhibit a bimodal distribution between high and low LSI species even at ambient temperature and pressure, but only in the inherent structure which is obtained by quenching to the nearest local minimum, i.e. when thermal excitations are removed. Also, it has

been shown ${ }^{114}$ that high and low LSI species correspond to LDL and HDL local structures. Here 
we will investigate the dependence of the spectral features on different cutoffs for characterizing the local structure in terms of these two extremes as described in the text.

However, the definition of the LSI is based only on the oxygen positions and thus does not reflect the presence or absence of H-bonds. XAS spectroscopy is sensitive to the presence of donated H-bonds, while the $1 b_{1}$ lone-pair non-bonding orbital seen in the emission spectroscopy participates in accepting H-bonds. In order to distinguish differently H-bond-coordinated water molecules, in addition to the LSI structural parameter, an H-bond criterion will be used. Here we use the cone-criterion developed by Wernet et al. ${ }^{6}$ based on XAS spectroscopy data. The H-bond cone criterion is a purely geometrical criterion where two water molecules are treated as H-bonded when the oxygen-oxygen distance $r$ is less than $r(\theta)$ defined as

$$
r(\theta)=r_{\max }-0.00044 * \theta^{2}
$$

where $\theta$ is the angle (degrees) between the internal $\mathrm{OH}$-bond and outer oxygen-oxygen distance and $r_{\max }$ is the cut-off distance; different $r_{\max }$ values may be used to select structures from the MD simulations with, e.g., very short and directional H-bonds or very asymmetrical with different $r_{\max }$ applied to define the structure. All threshold $r_{\max }$ values are specified in each case separately.

The liquid water model structures were obtained from path-integral molecular dynamics (PIMD) simulations with 32 beads. The opt-PBE-vdW functional ${ }^{116}$ PIMD simulations using 64 molecules and ambient conditions were obtained from ref. ${ }^{79}$ where the VASP code ${ }^{117}$ was used with projector-augmented wave potentials ${ }^{118}$, a plane-wave cutoff energy of $400 \mathrm{eV}$ and $\boldsymbol{k}$-point sampling limited to the $\Gamma$-point. PIMD trajectories at $298 \mathrm{~K}$ with 256 molecules and using the highly accurate MB-pol force-field which, in addition to the monomer, has been fitted to CCSD(T) calculations on a large set of dimers and trimers ${ }^{119-121}$ were supplied by F. Paesani ${ }^{122}$. 
All XES spectrum calculations have been performed using the Q-Chem software ${ }^{123}$ and clusters with 32 molecules extracted from the simulations and centered on the core-excited molecule. For the single snapshot calculations in Figure 1, in the case of the opt-PBE-vdW functional simulation with 64 molecules this meant 64 clusters with 32 molecules each, while for the MB-pol force-field trajectory 256 such clusters were extracted and computed. For Figures 2-4 when searching for criteria more than 7000 such clusters from steps along the trajectories were computed. Emission energies and oscillator strengths were computed using TDDFT ${ }^{124}$ within the Tamm-Dancoff approximation $^{125}$. The spectrum calculations have been performed in two steps in order to define the core-hole. First, a ground state calculation was performed on the water cluster where the central oxygen was replaced by fluorine and at the same time one electron was removed. Due to this, fluorine remained isoelectronic with oxygen. This allowed the desired oxygen $1 s$ orbital to be isolated, and the obtained orbitals were then used in the subsequent TDDFT calculation with a core-hole introduced in the relevant $1 s$ orbital (i.e. of the oxygen in the central molecule). The maximum overlap criterion $(\mathrm{MOM})^{126}$ was used in the TDDFT calculations in order to prevent collapse of the core hole during the SCF process. The XES transition values (oscillator strengths and energies) are obtained as negative eigenvalues in the TDDFT calculation. In order to get the continuous spectrum, the stick-like spectrum data were broadened using a Gaussian line shape with full width at half maximum (FWHM) $0.18 \mathrm{eV}$. As standard DFT functionals do not give reliable absolute transition energies ${ }^{127}$, the special functional $\mathrm{B}^{\mathrm{X}} \mathrm{LYP}$ was utilized which was optimized for core transitions ${ }^{99}$ (a comparison with CAMB3LYP is shown in the Supplementary Material (SM)). The parameter $X$ accounts for the amount of Hartree-Fock exchange, which in our case was set to 0.66 . Detailed information about the simulation technique and used DFT functional can be found elsewhere ${ }^{99}$. The water molecule for which XES has been computed was described using the IGLO-III basis set ${ }^{128}$ which was specially designed in order to improve the description of the core orbitals. The remaining water molecules in the cluster were described using the Pople 


\section{6-31G basis set ${ }^{129}$.}

The XAS spectra of the corresponding water clusters have been computed using TDDFT and the complex polarization propagator $(\mathrm{CPP})^{130-132}$ approach as implemented in the DALTON ${ }^{133}$ code, using the same computational set-up as in Ref. ${ }^{79}$. In all XAS calculations, the cluster size was restricted to 32 water molecules. Proper long-range Coulomb interaction between initial and final electronic states has been ensured by using the CAMB3LYP functional ${ }^{134}$ with modified parameters $\left(\alpha=0.19, \beta=0.81, \mu=0.33 \operatorname{Bohr}^{-1}\left(\mathrm{a}^{-1}\right)\right)^{135,136}$. A common lifetime broadening (half-width at half maximum) $\gamma=1000 \mathrm{~cm}^{-1}(0.124 \mathrm{eV})$ has been utilized in all CPP calculations. The basis set for the central water molecule in the cluster was chosen as IGLO-III ${ }^{128}[7 \mathrm{~s} 6 \mathrm{p} 2 \mathrm{~d}]$ for oxygen and IGLO-II [3s1p] for hydrogens. The remaining oxygen atoms were described via the MWB effective core potential $^{137}$ in order to ensure that only core excitations from the central water molecule were taken into account. The closest 6 water molecules to the central one were described using the triple- $\zeta$ IGLO-II basis set $^{128}$ for hydrogens, while the oxygens were described with a triple- $\zeta$ basis set obtained from the double- $\zeta$ MWB basis set by uncontracting the third PGTO in the first CGTO and adding a $d$-function with exponent 0.7 . For the rest of the water molecules in the cluster a double- $\zeta$ basis set $(2 s)^{138}$ was used for hydrogen and the MWB double- $\zeta$ basis set ${ }^{137}$ used for oxygen with the last $p$-function removed. In order to improve the description of the excited states, the basis set was augmented with additional diffuse functions placed at the central oxygen atom, this being a tailored $19 s 19 p 19 d$ basis set (even-tempered basis set with the first two exponents amounting to 1.238 and $0.884)^{139}$. Core-excitation energies computed with the TDDFT approach are usually too low in comparison with experiment due to the self-interaction error. For comparison with experiment all XAS spectra were thus shifted upward by $15 \mathrm{eV}$ in order to fit the experimental energy region.

\section{RESULTS AND DISCUSSION}


The non-resonantly excited XES spectrum of liquid water exhibits a double-peak structure in the lone-pair region as discussed above and shown in Figure 1. We have earlier demonstrated, for both liquid methanol ${ }^{108}$ and ethanol ${ }^{109}$ (which also exhibit a split in the lone-pair region ${ }^{140}$ ), and for models of water ${ }^{10,11,95,141}$, that the number of peaks and their positions are mainly determined by the initial structure while the width and relative intensity distribution is largely given by the corehole-induced dynamics which also depends on the initial structure. The sensitivity to different $\mathrm{H}-$ bond situations has also been demonstrated for various water clusters by Besley ${ }^{98}$ using equation of motion coupled cluster theory. Thus, it will be justified as a first step to compute static XES spectra for water structures to investigate the peak positions and how they correlate with the H-bond situations. This is also supported by a recent study ${ }^{110}$ of non-resonant XES, including core-holeinduced dynamics, on an MD model of water which found clear signatures of three broadened molecular valence states, but no new peaks or split arising from the dynamics, similar to earlier studies $^{11,103,141}$.

In Figure 1 we compare static XES spectra computed for all the molecules in a random snapshot in the PIMD simulations using either DFT with the opt-PBE-vdW ${ }^{116}$ van der Waals functional or the state-of-the-art MB-pol ${ }^{119-121}$ force-field of Paesani and coworkers. In the latter case a significantly larger box (256 molecules) could be used than in the ab initio DFT simulation (64 molecules) resulting in a significantly more smooth spectrum. We chose one random copy (bead) in each case for these simulations. We note that the involvement of the core-level makes XES a very local spectroscopy and the experimental spectrum can thus be regarded as a statistical sampling of the local environments of the core-ionized molecules ${ }^{96}$. We emphasize that the experimental spectrum, in spite of arising from a macroscopic number of molecules, does not correspond to an average, but to a sum of contributions from individual molecules in their instantaneous local 
environment. From a simulation point of view this corresponds to summing the individual contributions from all the molecules in the simulation box, assuming that the snapshot is representative of the statistics. Here we show one snapshot from each simulation for direct comparison; additional snapshots are in agreement with Figure 1 and are shown in the SM.

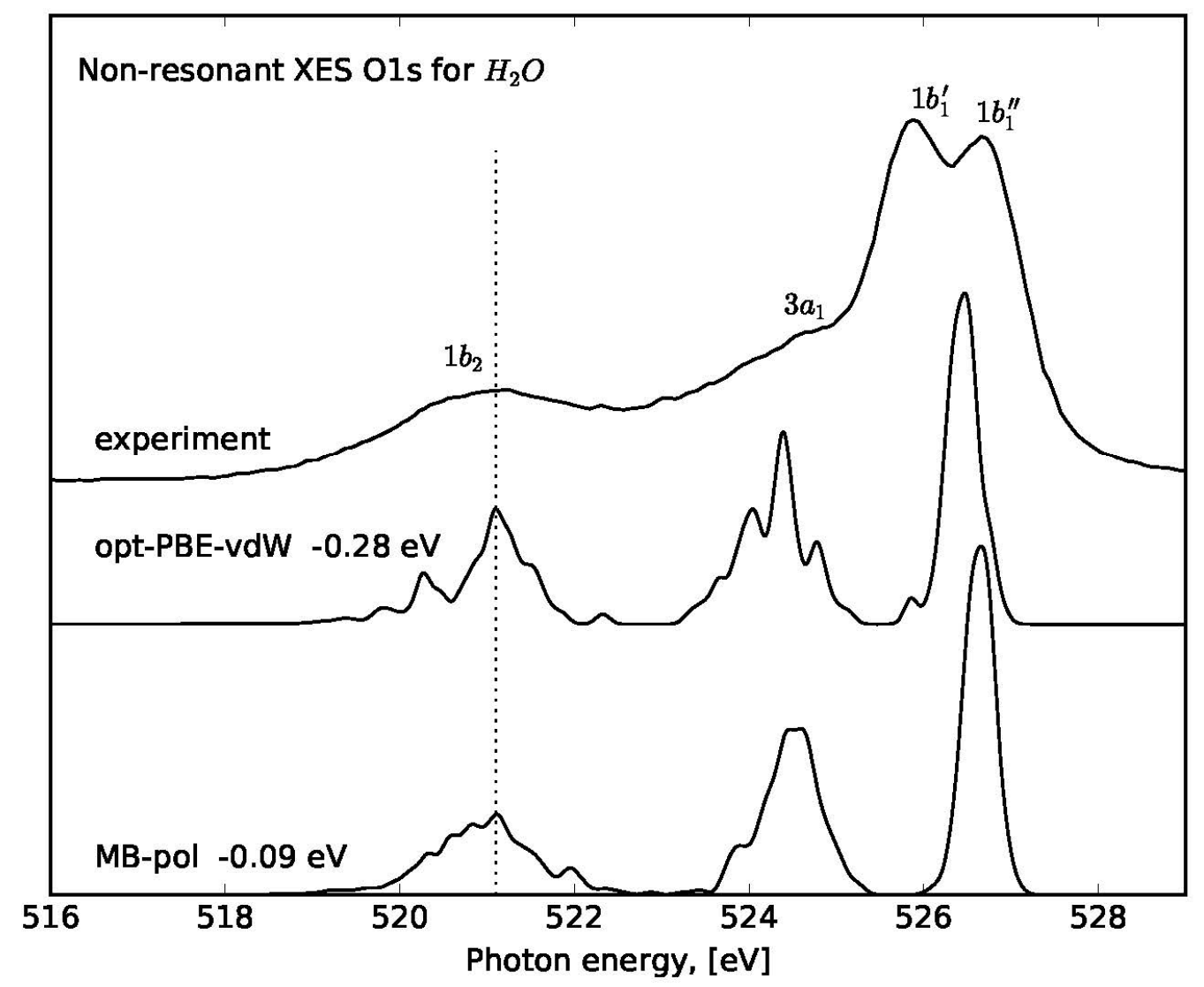

FIG. 1 X-ray emission spectra summed over all molecules in randomly selected PIMD snapshots and compared with the experimental spectrum from ref. ${ }^{11}$ (top). (Middle) XES spectrum for one bead of an opt-PBE-vdW snapshot containing 64 water molecules. (Bottom) XES spectrum computed for one bead of an MB-pol snapshot containing 256 water molecules. A shift of -0.28 and $-0.09 \mathrm{eV}$ has been applied for opt-PBE-vdW and MB-pol, respectively, to align with the $1 b_{2}$ feature in the experiment.

In the computed spectra the $1 b_{1}$ lone-pair peak shows up as a single peak in a position 
intermediate of the two peaks but more towards the higher emission energy peak in the experiment (note that a shift of -0.28 and $-0.09 \mathrm{eV}$ has been applied for opt-PBE-vdW and MB-pol, respectively, to align with the $1 b_{2}$ and $3 a_{1}$ features in the experiment). Apart from this, the character of the computed spectra shows good agreement with the experiment with the $1 b_{2}$ around $521 \mathrm{eV}$ and $3 a_{1}$ around $524.5 \mathrm{eV}$. It should be noted that the neglect of dynamics, or vibrational interference ${ }^{142}$, leads to spectral features that are too sharp compared to experiment. We emphasize that the two split peaks have been shown experimentally to both be of $1 b_{1}$ symmetry ${ }^{93,94}$ and that inclusion of core-hole-induced dynamics in spectrum simulations based on current MD structures

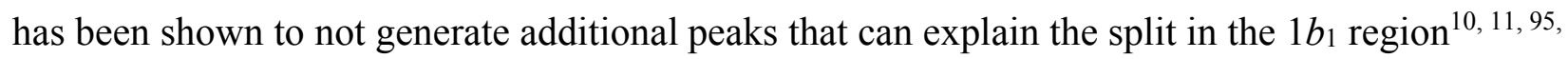
96, 104, 108-110. The $1 b_{1}$ peak position has been shown to depend on the H-bonding situation ${ }^{98,110}$ which we explore below. 

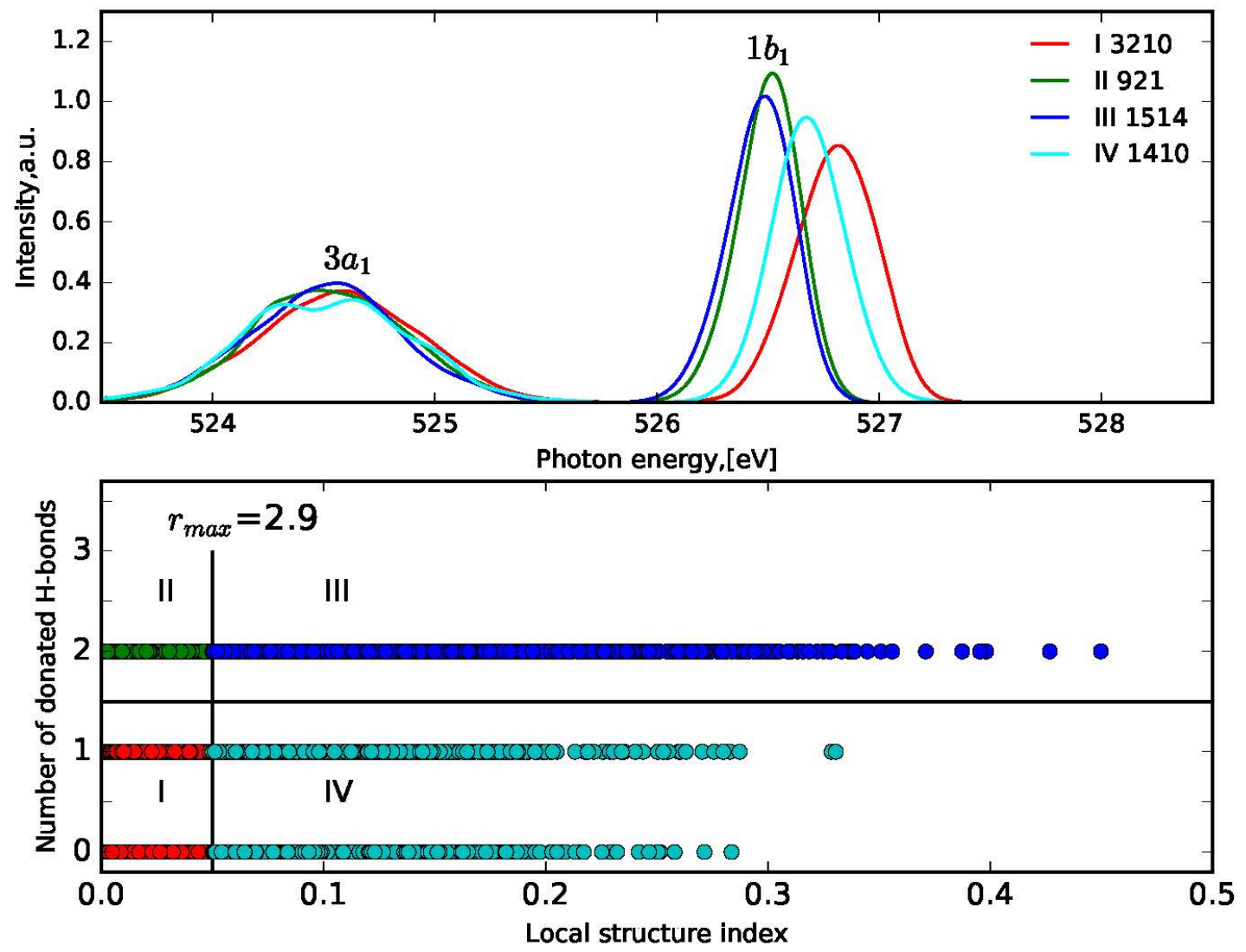

FIG. 2 Donated H-bonds and LSI parameter. (Bottom) Subdivision of structures according to the selected order parameters with LSI parameter on the horizontal axis and number of donated H-bonds $\left(r_{\max }=2.9 \AA\right)$ on the vertical axis. The four different classes are defined according to the straight lines in the figure and the number of instances drawn in each class is indicated in the legend. (Top) Computed XES for the four different classes showing the $3 a_{1}$ around $524.5 \mathrm{eV}$ and the $1 b_{1}$ appearing in the range 526 to $527 \mathrm{eV}$ depending on the class.

XAS has been shown to be sensitive to the local H-bonding where molecules with asymmetrically donated H-bonds, i.e. one well-defined and one weak or broken, contribute to the

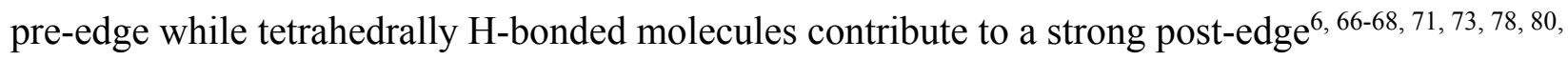
${ }^{143}$. The LSI parameter gives complementary information in terms of the structure of the O-O pairdistribution function where very low values are found for an HDL-like local environment and high 
values correspond to an LDL-like environment ${ }^{114}$. Here we will use these two parameters, the number of donated or accepted H-bonds (within a cutoff at $2.9 \AA$; other cutoffs in the range 3.0$3.2 \AA$ give very similar results and are shown in the SM) and the LSI parameter, to classify structures from the MD simulations and search for correlations between these parameters and the $1 b_{1}$ position in the corresponding XES spectrum. The classification using donated H-bonds is shown in Figure 2 together with the computed peak position for each class; the statistics are good where the peak for class I is the sum of 3210 individual spectra, as indicated in the inset, while classes II-IV contain 921, 1514, and 1410 spectra, respectively, from structures representing these classes. In Figure 3 we show the corresponding classification of the same structures as in Figure 2 using instead the number of accepted H-bonds.
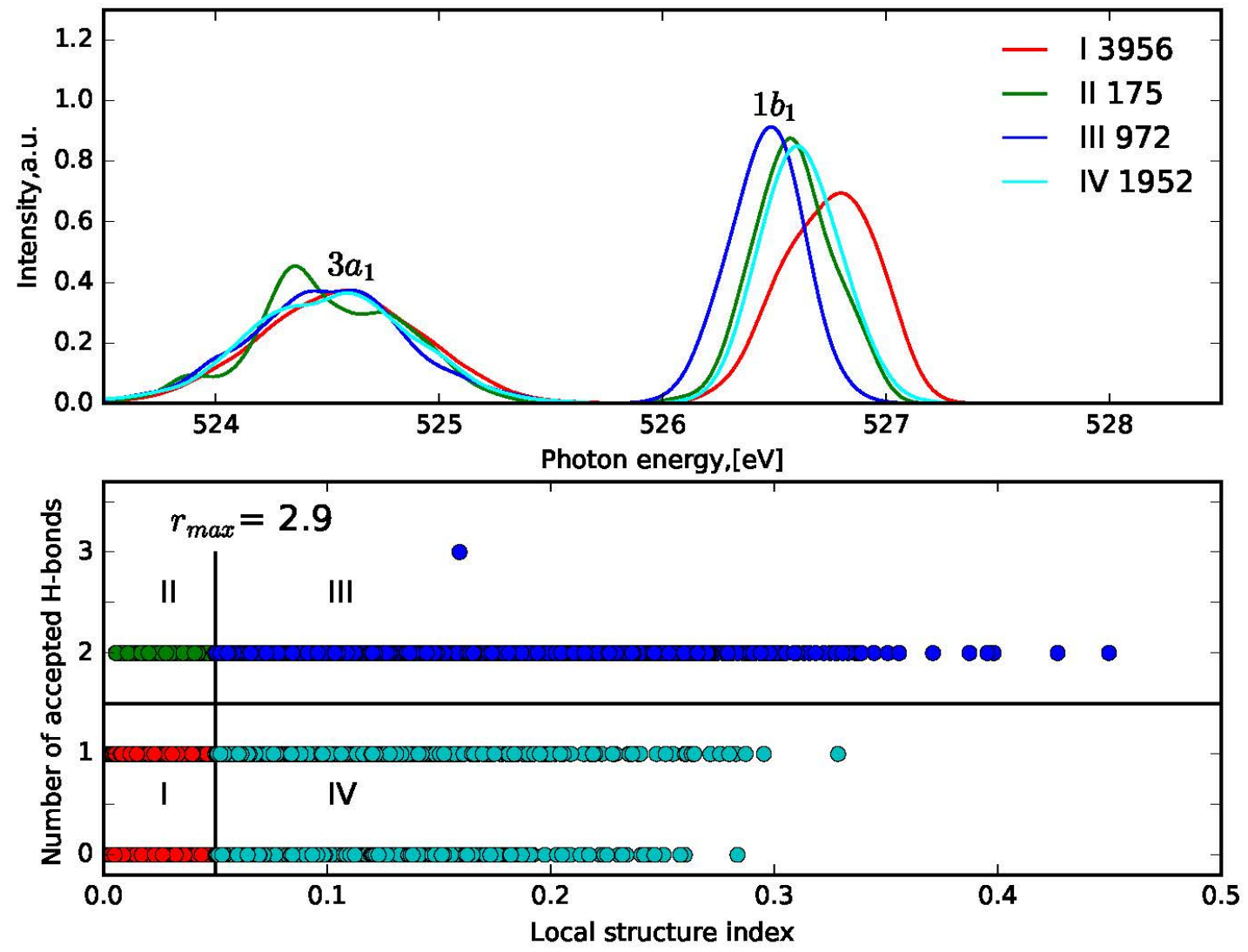

FIG. 3 Accepted H-bonds and LSI parameter. (Bottom) Subdivision of structures according to the selected 
order parameters with LSI parameter on the horizontal axis and number of accepted H-bonds $\left(r_{\max }=2.9 \AA\right)$ on the vertical axis. The four different classes are defined according to the straight lines in the figure and the number of instances drawn in each class is indicated in the legend. (Top) Computed XES for the four different classes showing the $3 a_{1}$ around $524.5 \mathrm{eV}$ and the $1 b_{1}$ appearing in the range 526 to $527 \mathrm{eV}$ depending on the class.

From Figure 2 we observe a clear dependence in the computed peak positions on the selected structural order parameters where very disordered, HDL-like, species with asymmetric donated Hbonds (low LSI value, one donated H-bond; class I) lie at the highest energy while very tetrahedral, LDL-like, species with two well-defined donated H-bonds (high LSI value, two donated H-bonds; class III) together with species of class II (two well-defined H-bonds and low LSI value) lie at the lowest emission energy with class IV (one well-defined donated H-bond and high LSI value) in between. From Figure 3, where we investigate the dependence on accepted H-bonds, we again find the largest separation between classes I and III, but now with both classes II and IV in between these extremes. Thus the number of $\mathrm{H}$-bonds seems to be the more significant parameter in determining the $1 b_{1}$ position. This is fully consistent with an earlier assignment of the two peaks in the experiment ${ }^{9-11,96,141}$ and the recent study by Shen et al. ${ }^{110}$ and is further analyzed in Figure 4, which collects the computed spectra according to H-bonding situation irrespective of the LSI value (using other cutoffs in the range 3.0-3.2 $\AA$ give very similar results and are shown for Figures 
2-4 in the SM).
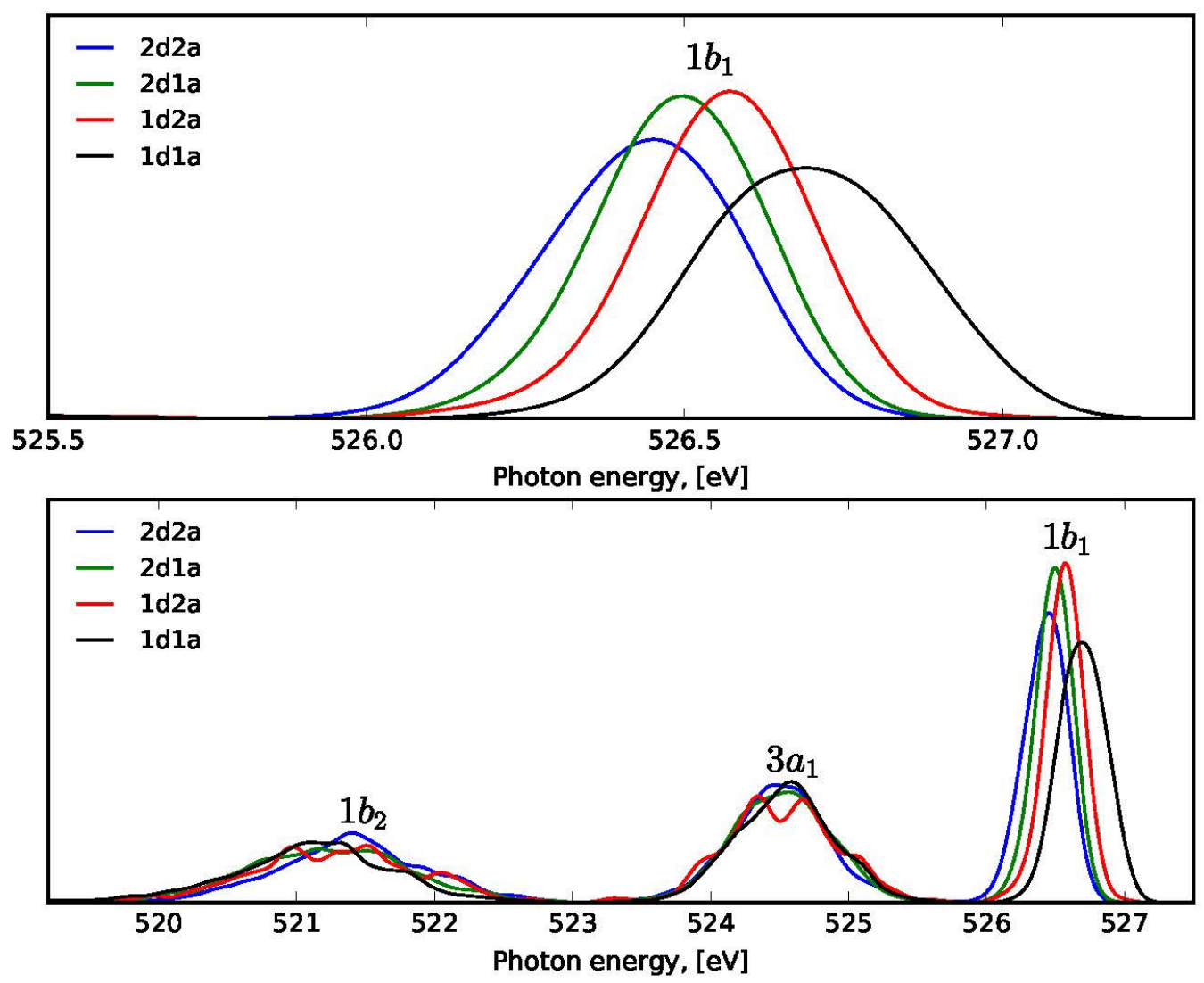

FIG. 4 Classification of structures according to the number of accepted and donated H-bonds $\left(r_{\max }=2.9 \AA\right)$ as double-donor/double-acceptor (2d2a), double-donor/single-acceptor (2d1a), single-donor/doubleacceptor (1d2a) and single-donor/single-acceptor (1d1a). (Top) Computed $1 b_{1}$ position for each class. (Bottom) Overall spectra in the valence region for each class.

From Figure 4 we note that, based on the H-bond criterion, the more than 7000 sampled and computed structures clearly separate into four different classes in terms of the position of the $1 b_{1}$ peak, with local structures that are unbalanced in terms of number of donated and accepted $\mathrm{H}$ bonds contributing intermediate between the $2 \mathrm{~d} 2 \mathrm{a}$ and $1 \mathrm{~d} 1 \mathrm{a} \mathrm{H}$-bond-balanced structures (other $r_{\max }$ values give similar results; see SM). This would correspond to the region between the two peaks observed experimentally and we conclude that these situations cannot occur frequently in the real 
liquid since this would result in a single broad peak in the $1 b_{1}$ region. We also note that the splitting between the maxima of the $2 \mathrm{~d} 2 \mathrm{a}$ and $1 \mathrm{~d} 1 \mathrm{a}$ distributions is significantly smaller than in the experiment, but the width of the peaks is large enough to support a larger split for subclasses of these species.

We observe further that the computed positions of the $1 b_{2}$ peak at $521.5 \mathrm{eV}$ and the $3 a_{1}$ peak at $524.5 \mathrm{eV}$ are very similar for all four classes which excludes an earlier interpretation of the split in the lone-pair region in terms of the initial core-hole state in the XES process ${ }^{96}$ and instead indicates a final state effect. We note here that the $\sim 1.4 \mathrm{eV}$ width of the valence XPS $1 b_{1}$ peak $^{101}$, 102, 144 can indeed support the observed split in XES when one also takes into account the proposed dominance of the HDL species, i.e. the peak at high emission energy.

While the peaks are resolved in XES due to the similar screening response from the environment to the charge in the core and in the valence, they are unresolved in XPS due to the strong response to the ionization process giving rise to intermolecular vibrations ${ }^{96}$. With the present results for the split in the lone-pair region, while the $1 b_{2}$ and $3 a_{1}$ are unaffected, it seems clear that the split is a final state effect, where the lone-pair valence-hole (nominal positive charge) is localized mainly on the initially core-excited molecule and is sensitive to the number of (positively charged) protons $\mathrm{H}$ bonding to it as well as to the H-bond donation. Thus, where XAS is sensitive to the donated Hbonds the molecule is involved in, XES is instead more sensitive to the total number of H-bonds.

In Figure 5 we illustrate the interpretation of the split through a qualitative energy level diagram and the resulting emission energies as the system relaxes, indicated through the emission spectrum on the right. The relative energies of the different final hole states are indicated with the $1 s^{-1}$ corehole state at the highest energy, followed by the $1 b_{2}{ }^{-1}$ and $3 a_{1}{ }^{-1}$ hole states and, finally the $1 b_{1}^{-1}$ state. From an electrostatic viewpoint, the creation of a hole in a valence orbital is equivalent to adding a 


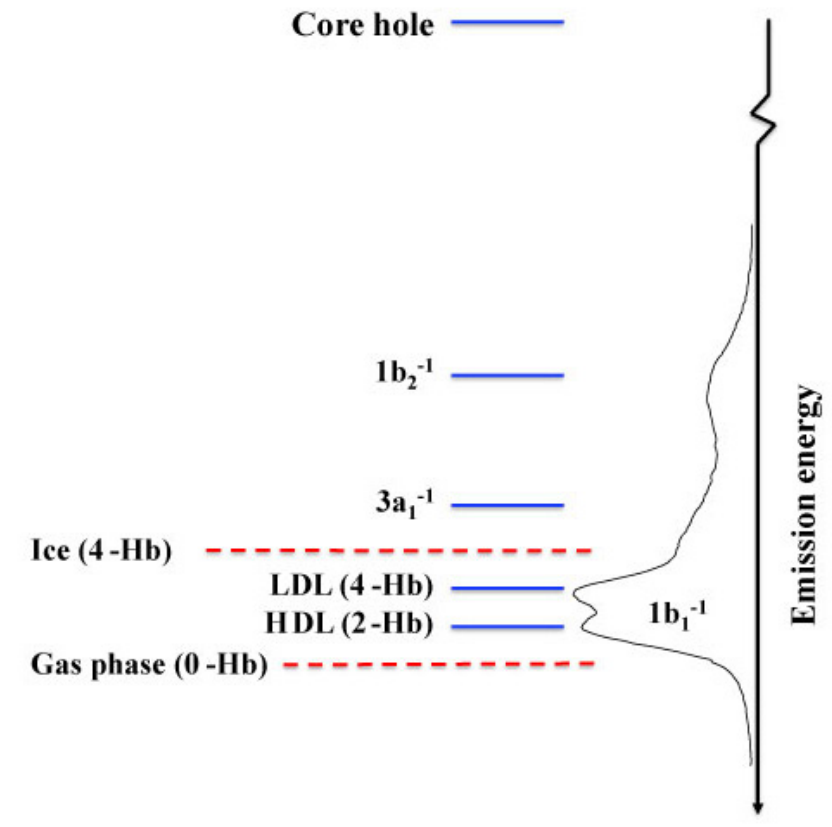

Fig. 5 Schematic illustration of relative energies of the core-hole and the valence-hole states involved in the XES of water. The decay is from the high-energy core-hole state to the various valence-hole states. The $1 b_{1}^{-1}$ positions of gas phase water with no $\mathrm{H}$-bonds $(0-\mathrm{Hb})$ and crystalline ice with two accepted and two donated H-bonds $(4-\mathrm{Hb})$ are indicated with dashed lines. The $1 b_{1}^{-1}$ final state of molecules in HDL local structures with only one accepted and one donated H-bond is less destabilized than for molecules in tetrahedral LDL local environments with four $\mathrm{H}$-bonds interacting unfavorably with the created valence hole. positive charge to the original orbital, which changes the electrostatic interaction if the orbital is localized and directly interacting with charges on neighboring molecules. The $1 b_{2}$ and $3 a_{1}$ bonding orbitals are polarized towards the oxygen and thus less sensitive to the environment; the unoccupied antibonding $2 b_{2}$ and $4 a_{1}$, on the other hand, are polarized towards the hydrogens, which gives the sensitivity to H-bond donation in XAS. For the localized lone-pair $1 b_{1}$ orbital the situation is different, since it directly interacts with the partial positive charge from $\mathrm{OH}$-groups on neighboring molecules when accepting H-bonds. This leads to an unfavorable interaction and increased energy

for the $1 b_{1}^{-1}$ state the more H-bonds that are accepted. Thus, as indicated in Figure 5, the lone-pair $1 b^{-1}$ state in gas phase water will be at the lowest total energy (and thus the highest emission energy), while one, two, three and four H-bonds will stepwise raise the energy of the $1 b^{-1}$ state with the greatest destabilization (and consequently lowest emission energy) in the case of crystalline ice, as indicated in the figure. Based on the observed well-defined split between the two situations, real water thus seems to prefer paired donated and accepted H-bonds, i.e. either single-donor/singleacceptor or double-donor/double-acceptor configurations, as also found from quantum chemical studies where maximum stability in individual H-bonds is obtained when accepted and donated Hbonds are paired ${ }^{145}$. 
We now turn to a comparison of XES and XAS for classes I and III, i.e. respectively very disordered and very tetrahedral. The selection was based on the structure of the H-bond environment and LSI values as deduced from the discussion of Figures 2-4 and earlier studies of XAS on water ${ }^{6,71}$. The central water molecule in each tetrahedral cluster has four strong H-bonds which have been obtained within the cone criterion using rmax $=2.9 \AA$. The LSI values in the tetrahedral clusters lie between 0.1 and 0.29 . The asymmetric clusters are characterized by an asymmetric H-bond situation where the central water molecule has only one strong accepted and one strong donated H-bond. The LSI values in the asymmetric structure set lie in the range between 0.001 and 0.012. These structural sets were extracted from the opt-PBE-vdW PIMD trajectory, but similar local structures could equally well have been extracted from the MB-pol snapshots.
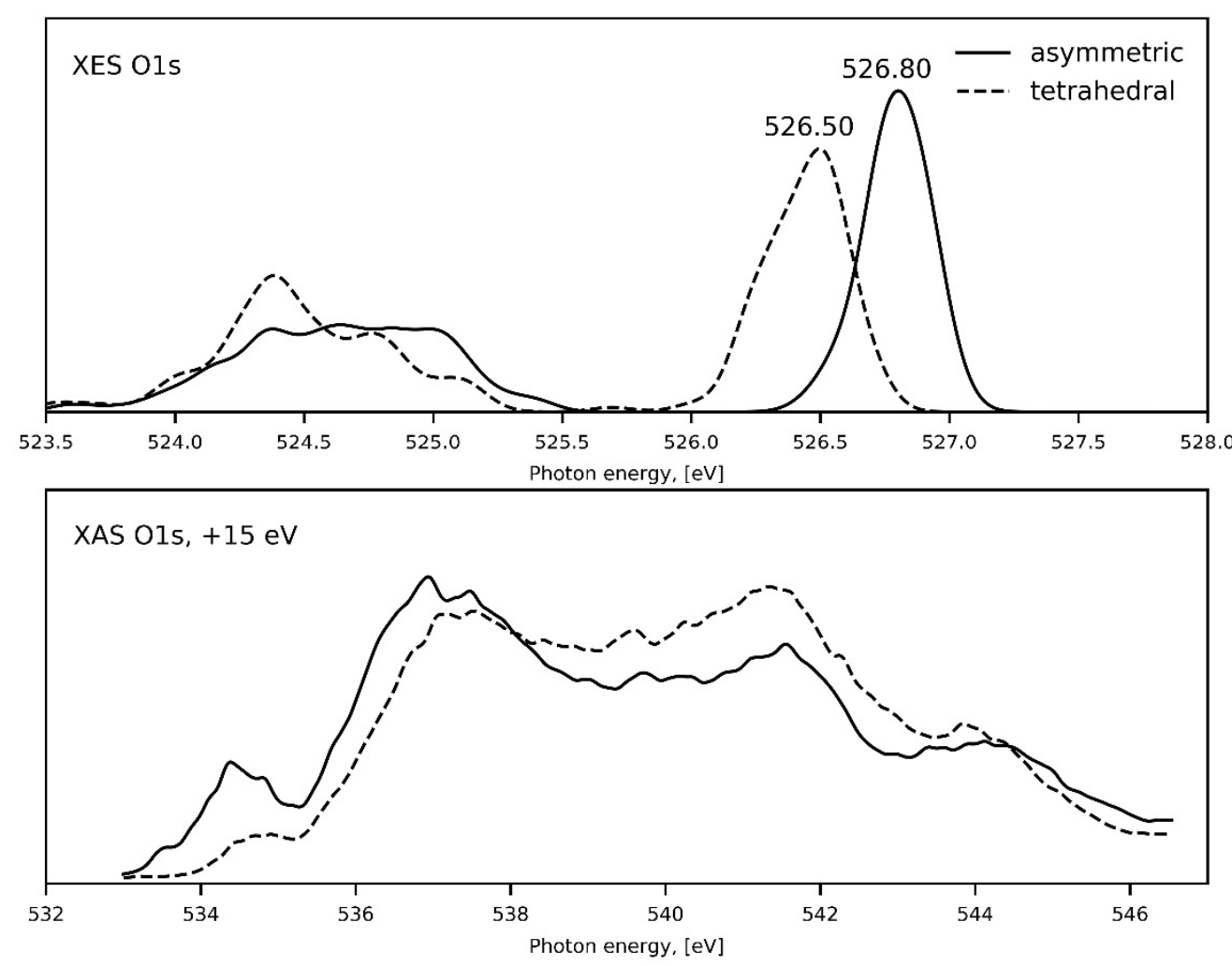

FIG. 6 XES (top) and XAS spectra (bottom) obtained for the tetrahedral (dashed) and asymmetric (solid) structure 
sets.

In Figure 6 we show the resulting XES and XAS spectra for these two classes with a clear split between the resulting $1 b_{1}$ peaks with the asymmetric (Class I) lying at higher emission energy. In XAS we find a well-defined and sharp pre-edge peak at $534-535 \mathrm{eV}$ and enhanced main-edge near $537 \mathrm{eV}$ from the asymmetric structures in agreement with earlier studies, $59,66,67,71,73,143$. The computed post-edge intensity is weaker than for tetrahedral molecules, but still sharp and with significant intensity, which may indicate that a broader range of donated H-bonds should be considered ${ }^{146}$. The tetrahedral molecules (Class III) show only weak intensity at the pre-edge while the post-edge around $541 \mathrm{eV}$ is enhanced compared to the asymmetric species. This thus connects the two spectroscopies structurally, as has been done previously in experiment ${ }^{9,}{ }^{66}$, and puts additional constraints on local structures in the liquid. However, the split between the two peaks in $\mathrm{XES}, 0.3 \mathrm{eV}$, is too small compared with experiment $\left(0.8-0.9 \mathrm{eV}\right.$, depending on temperature $\left.{ }^{11}\right)$. Core-hole-induced dynamics is not included here, but this should shift the high-emission energy peak slightly towards lower energy as observed from experimental comparison of $\mathrm{H}_{2} \mathrm{O}$ and $\mathrm{D}_{2} \mathrm{O}$ and also in earlier simulations ${ }^{10,11}$.

We note that the computed $1 b_{1}$ peak of the tetrahedral species is quite broad and asymmetric towards lower emission energy which can indicate that additional structural constraints could enhance the split towards the experimentally observed. In Figure 7 we investigate the effects of Hbond distance on the spectra within each class with either short or long H-bonds for the two classes. For the tetrahedral species we define long bonds as all H-bonds longer than $2.75 \AA$ and short bonds as all H-bonds shorter than $2.68 \AA$. For the asymmetric species long H-bonds are selected with all bonds longer than $2.8 \AA$ and for the short bonds the H-bond distance is taken as shorter than 2.65 $\AA$. With the first peak in the O-O pair-distribution function at $2.8 \AA$ and onset at $2.5 \AA^{147}, 2.68 \AA$ still corresponds to a significant fraction of the first peak in the distribution of nearest-neighbor distances. We find a distinct sensitivity to the H-bond distance in the two classes. 

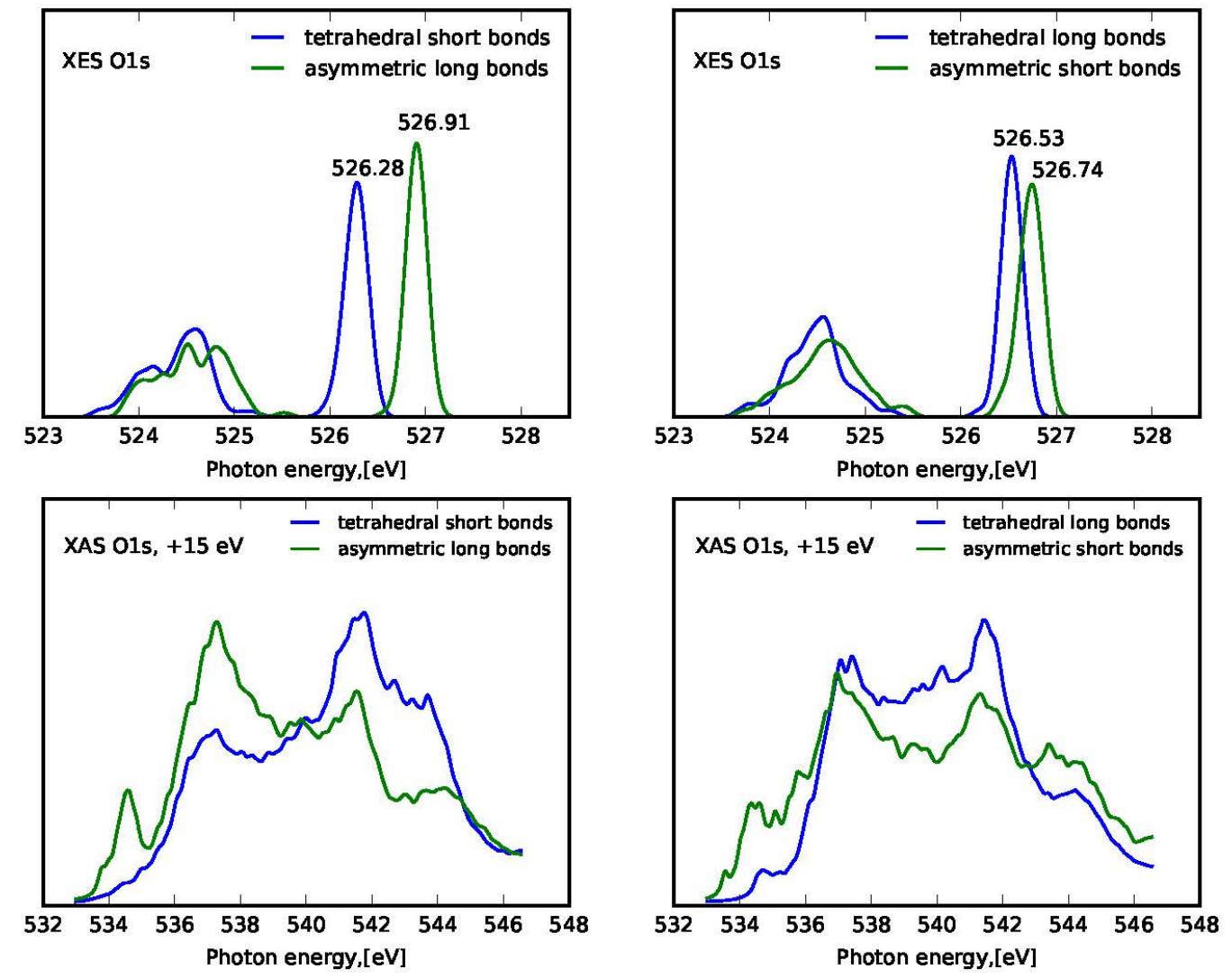

FIG. 7 Effects of H-bond distance on computed XES (top) and XAS (bottom) spectra within each class of structures. (Left) Short H-bonds $\left(r_{\max }=2.68 \AA\right)$ for the tetrahedral species and long H-bonds $\left(r_{\min }=2.8 \AA\right)$ for the asymmetric species. (Right) Long H-bonds $\left(r_{\min }=2.75 \AA\right)$ for the tetrahedral species and short H-bonds $\left(r_{\max }=2.65 \AA\right)$ for the asymmetric species.

With long H-bonds for the tetrahedral species and short bonds for the asymmetric ones (Figure 7, right panels) we find a split in XES of $0.21 \mathrm{eV}$ and also much of the characteristic structure in XAS is lost. The intensity in the pre-edge region is still enhanced for the asymmetric species, but the main-edge is now dominated by tetrahedral species in contradiction to what is observed from water excited resonantly at the main-edge of XAS, for which the high-emission energy peak (asymmetrical species) is enhanced in $\mathrm{XES}^{9}$. For the case of tetrahedral species with short H-bonds and long H-bonds for the asymmetric species we find a split of $0.62 \mathrm{eV}$ in XES and a significantly 
more well-defined XAS spectrum with sharp pre-edge and main-edge contributed by the asymmetric species and very weak pre-edge and high and broad post-edge intensity contributed by the tetrahedral species. In Figure 8 we focus on the H-bond distance-dependence of the split between the two peaks in XES.

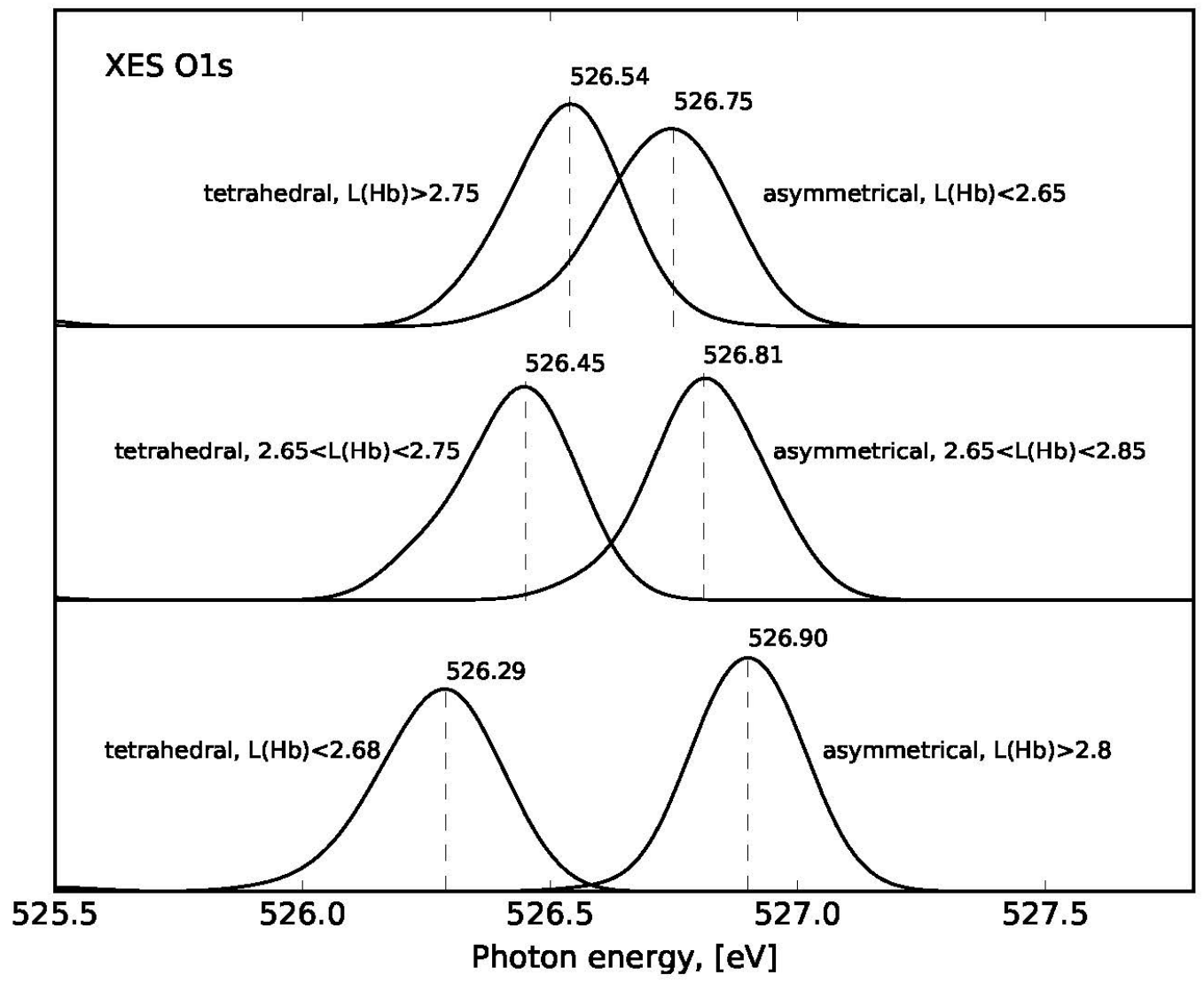

FIG. 8 XES spectra for the tetrahedral and asymmetric structure sets with additional restrictions on the H-bond lengths. (Top) Tetrahedral structures with long $(>2.75 \AA)$ and asymmetric structures with short $(<2.65 \AA)$ H-bonds. (Middle) Tetrahedral with H-bonds in the range 2.65 to $2.75 \AA$ and asymmetric with H-bonds in the range 2.65 to $2.85 \AA$. (Bottom) Tetrahedral with short $(<2.68 \AA)$ and asymmetric with long $(>2.8 \AA)$ H-bonds. The peak positions are given in $\mathrm{eV}$ for each spectrum.

As in the previous case, the central water molecule has two strong donated and two strong accepted H-bonds in the tetrahedral clusters, and one strong accepted and one strong donated $\mathrm{H}-$ 
bond in the asymmetric clusters. The LSI values for the tetrahedral clusters lie in the range between 0.15 and 0.45 , and for the asymmetric clusters between 0.002 and 0.012 . We find a clear dependence on the H-bond distance, where tetrahedral, LDL-like, species should have H-bond distances on the short side of the O-O nearest neighbor peak ${ }^{147}$ while the asymmetric, HDL-like, should contribute to the longer distances in the first peak including the interstitial positions giving intensity between the first and second correlations of the O-O pair-distribution.

That tetrahedral species should contribute mainly to the correlation at shorter distances in the O-O pair-distribution is fully consistent with extended X-ray absorption fine-structure (EXAFS) measurements on liquid water ${ }^{148,149}$ which resulted in a first peak at short (2.70 $)$ O-O separation and peak height 3.2. This should be compared to the benchmark X-ray diffraction (XRD) O-O pairdistribution function of Skinner et al. ${ }^{147}$ where the peak position is at $2.80 \AA$ and height 2.57 . The discrepancy between EXAFS and XRD was resolved by Wikfeldt et al. ${ }^{150}$ who generated a range of structural models from which a local pair-distribution contribution could be obtained and the corresponding EXAFS signal computed. Using SpecSwap ${ }^{151}$ reverse Monte Carlo (SpecSwapRMC) simulations to simultaneously fit the pair-distribution function from XRD and the measured EXAFS signal, a structural solution was found which required a subset of tetrahedral structures with very short and directional H-bonds ${ }^{150}$. The complementary information in XRD and EXAFS is due to the fact that $\mathrm{XRD}$ is mainly sensitive to $\mathrm{O}-\mathrm{O}$ correlations, but rather insensitive to the presence of $\mathrm{H}$-bonds ${ }^{152}$, while EXAFS is very sensitive to short and directional H-bonds due to the enhanced backscattering of the emitted electrons from the focusing effect of a positively charged hydrogen between the emitting oxygen and the scattering neighboring oxygen to which it forms an H-bond. The signal is further enhanced if the nearest neighbors are well-ordered, as in tetrahedral coordination, making EXAFS see mainly this component while XRD is less sensitive to disorder and H-bonding. Short H-bond distances, around and below $2.7 \AA$, for the tetrahedral, LDL-like, component in ambient water are thus fully consistent with XRD, EXAFS, XAS and XES. 


\section{CONCLUSIONS}

Here we investigate requirements on the H-bond network in simulated water to simultaneously give agreement between computed and experimental XAS and XES spectra. We show that, if one computes XES for all molecules of a snapshot of simulations using the currently best models, the resulting spectrum exhibits only one peak, which furthermore is intermediate between the two split peaks in the experiment. In these calculations we neglect dynamical or vibrational interference effects $^{142}$ which have significant effects on peak intensities, but have been shown to not generate additional features in the H-bonded cases investigated so far ${ }^{95,104,108}$. In a recent study of XES on water based on MD simulated structures, the effects of core-hole-induced dynamics were also included and also in this case there were no extra peaks induced by the dynamics ${ }^{110}$. The spectra were found to be sensitive to the number of H-bonds with molecules with broken H-bonds contributing at higher emission energy similar to what is found here. Since only a small fraction (9.375\%) of the molecules in the simulation was reported to have broken H-bonds ${ }^{110}$, the conclusion must also in this case be that summing spectra from all the molecules in the simulation will only give one $1 b_{1}$ peak and not the split observed in experiment; this seems also to be the case from fig. 1 in ref. ${ }^{110}$.

We note further that the spectra including core-hole-induced dynamics in ref. ${ }^{110}$ exhibit unphysical intensity at higher emission energy than the $1 b_{1}$ peak. This seems to arise from a continuous shift of the spectra to higher energy along the trajectory in time. This is not in agreement with experiment where the $1 b_{1}$ peak at highest emission energy in $\mathrm{H}_{2} \mathrm{O}$ is shifted to lower energy than for $\mathrm{D}_{2} \mathrm{O}^{11}$. We have earlier shown that, for a proper description of the core-hole-induced dynamics of the $\mathrm{OH}$-group using classical MD, one needs a sufficient sampling of both $\mathrm{OH}$ positions and proton momenta from the quantum probability distribution as initial conditions ${ }^{95,104,108,109}$. For an individual $\mathrm{O}-\mathrm{H}$ stretch mode a minimum of two $\mathrm{OH}$ positions and two proton momenta (times 
two to account for the initial zero expectation value), i.e. 8 initial conditions, has been found sufficient $^{108,109}$. Each sampled structure in water will thus give rise to 64 trajectories, each of length $40 f_{s}$ with more than 150 spectra from each trajectory, i.e. around 10000 spectra for each molecule that is selected which should be weighed together including the important life-time vibrational interference effects ${ }^{95,104,142}$. Before embarking on such a task it is necessary to ensure that the initial MD structures that are used are representative of the situation in real water.

To this end we apply two criteria, the local structure index (LSI) ${ }^{113}$ and number of H-bonds defined according to a cone-criterion ${ }^{6}$, to classify local structures and show that the $1 b_{1}$ lone-pair peak position is sensitive to both the number of $\mathrm{H}$-bonds and to the tetrahedrality of the environment, as measured by the LSI. We demonstrate further that the magnitude of the split between the two peaks depends on the H-bond distances, where short $(<2.7 \AA)$ and well-defined H-bonds characterize the tetrahedral or low-density-like component in the liquid while the disordered or high-density-like component requires asymmetric donated H-bonds, which are furthermore elongated. The spectral features from both XAS and XES are thus consistent with a bimodal distribution of local structures in real water resulting from fluctuations between two dominating local structural environments. Simulated water seems to give a structural average of these fluctuating species, where instead our interpretation and simulation of the experimental spectra indicate that they should be structurally well separated. We provide a simple understanding of the split in terms of the difference in electrostatic interaction when the created localized $1 b^{-1}$ state participates in $\mathrm{H}$-bonds. We demonstrate that structures that can represent the experimental spectroscopic features are present in the MD simulations, but they are rare in spite of the simulations reproducing well the experimental $\mathrm{O}-\mathrm{O}$ radial distribution function.

The proposed bimodal structural distribution in terms of local H-bonding is fully in accordance with the measured $\mathrm{OH}$-stretch frequency distribution, as shown, e.g., by recent resonant XES highresolution measurements of participator decay ${ }^{153}$, i.e. where the excited electron falls back to the 
ground state with energy loss corresponding to vibrational excitations in the ground state potential. Exciting at the XAS pre-edge, corresponding to excitation into electronic $\mathrm{OH}$ antibonding $\sigma^{*}$ states localized $^{67,154}$ on weakly H-bonded $\mathrm{OH}$ oscillators gives a frequency close to gas phase ${ }^{112,153}$ while excitation at the post-edge, which is due to strong H-bonds, gives a red-shifted frequency ${ }^{153}$. Detuning the excitation energy to below the pre-edge in XAS the resulting XES becomes nonselective and samples the distribution of oscillators according to the statistical distribution of local arrangements in the liquid and the optical Raman spectrum is obtained ${ }^{153}$. Since excitation at the pre-edge is uniquely correlated with the high-emission-energy peak in $\mathrm{XES}^{9,96}$, this provides strong evidence for the existence in real water of distinguishable local H-bonding environments ${ }^{3,14}$.

Lawrence and Skinner have in a series of papers analyzed the sensitivity of the $\mathrm{OH}$-stretch to the H-bonding environment ${ }^{155-158}$, mainly focusing on the decoupled OH (OD) stretch of HDO in $\mathrm{D}_{2} \mathrm{O}\left(\mathrm{H}_{2} \mathrm{O}\right)$ based on a correlation between the frequency and the electric field along the stretch coordinate and reporting the difference between $\mathrm{H}$-bonded and non-H-bonded $\mathrm{OH}$ in terms of frequency. Based on the same correlation Leetmaa et al. ${ }^{152}$ showed that both highly tetrahedral and highly asymmetric structural solutions are consistent with X-ray and neutron diffraction data as well as the Raman spectrum for $\mathrm{HDO}$ in $\mathrm{D}_{2} \mathrm{O}$. It should, however, be noted that the isotope substitution localizes the $\mathrm{OH}$ oscillator, as intended, but eliminates the collective vibrations that are particularly important for the proposed LDL component ${ }^{3}$ and may also not provide an unbiased sampling of structures in the liquid $^{112}$.

The MB-pol force-field is arguably the most ambitious classical force-field to date, but is still found to give only one peak in the computed XES, which is dependent on the local H-bonding structure. This would seem to be at odds with the good agreement that has been reported for computed IR and Raman spectra using this model ${ }^{5,42}$; these spectroscopies also depend on the local H-bonding structure. In spite of great success in reproducing the IR spectrum at lower frequencies, there are, however, still discrepancies with experiment in terms of the OH-stretch band in both the 
computed IR and Raman spectra ${ }^{42,159,160}$. In both cases the spectra are too narrow and lack intensity particularly in the low-frequency region which is derived from collective vibrations involving tetrahedral LDL-like species $^{3,38}$; the temperature dependence of this peak ${ }^{39}$, as well as the measured polarization dependence ${ }^{38}$, makes an interpretation of the low-frequency region of the $\mathrm{OH}$-stretch in terms of a Fermi resonance unlikely. In Figure 9 we make a direct comparison of unpolarized Raman spectra from experiment ${ }^{3,161,162}$ and classical MD simulated spectra from ref. ${ }^{42}$. The computed Raman spectrum in the $\mathrm{OH}$-stretch region using $\mathrm{MB}-\mathrm{pol}^{42}$, notwithstanding that this represents the current state-of-the-art, shows only a broad, featureless peak which continuously shifts toward lower frequency with decreasing temperature ${ }^{42}$.
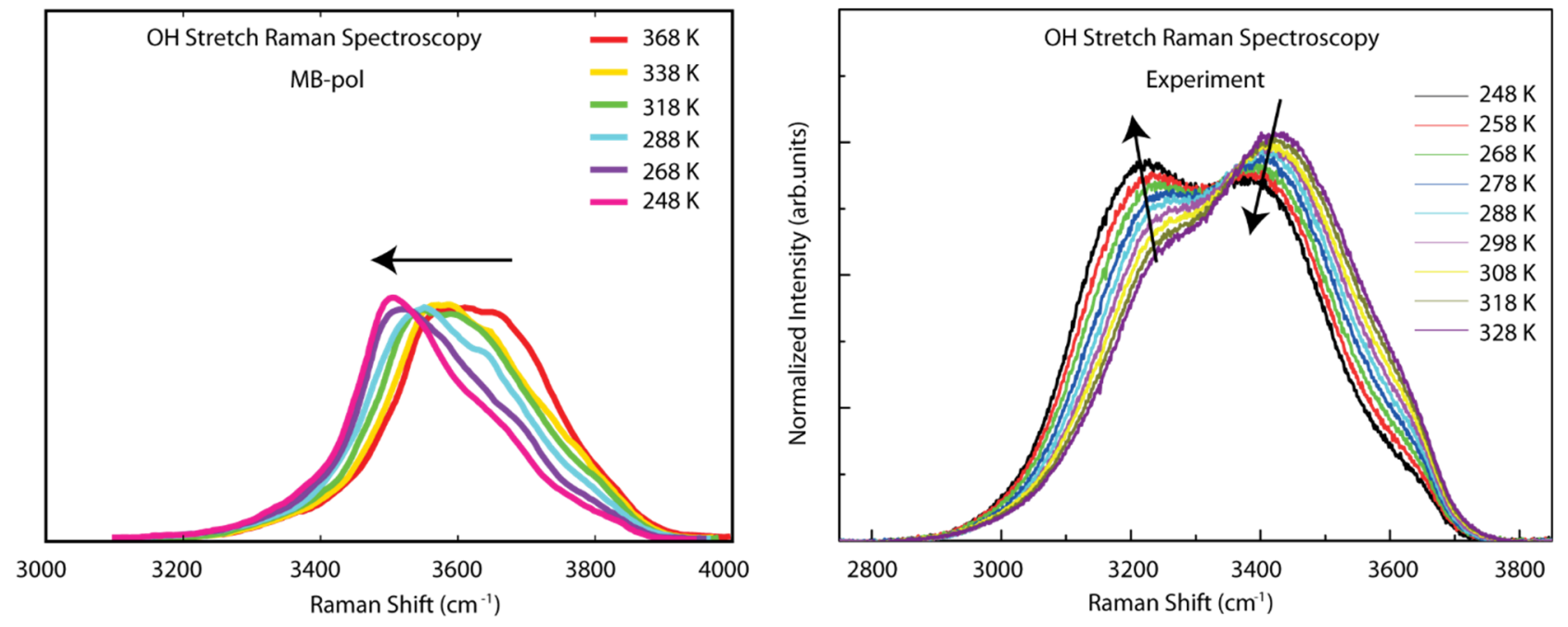

FIG. 9 Comparison of the temperature dependence of unpolarized Raman spectra for the $\mathrm{OH}$-stretch band of $\mathrm{H}_{2} \mathrm{O}$ water as obtained by (left) classical MB-pol simulations ${ }^{42}$ and (right) experiment ${ }^{3,162}$; arrows indicate shifts of intensity with decreasing temperature. The simulation (left) shows only a single broad feature shifting towards lower frequency with decreasing temperature in agreement with the picture obtained here from our XES calculations that the simulation gives an average of structures. Experiment (right) exhibits transfer of intensity from the high-frequency side to the lowfrequency side with decreasing temperature without significant frequency shifts, which, from the sensitivity of the $\mathrm{OH}-$ stretch to the environment, is better explained in terms of temperature-dependent changes in the distribution of fluctuations between two dominating local structural environments as concluded from the computed XES.

Experimentally a two-peak spectrum is observed with intensity transferred from the blue-shifted peak to that on the red-shifted side with decreasing temperature ${ }^{3,161,162}$. Thus, also the vibrational 
spectroscopies, as obtained from the state-of-the-art MB-pol simulations, seem to represent an average of the two structures indicated by the X-ray spectroscopies. This leaves room for improvement, but the origin of this discrepancy between simulated and real water is presently unknown.

\section{SUPPLEMENTARY MATERIAL}

The supplementary material contains a comparison of spectra using BxLYP as in the main paper and CAMB3LYP, additional snapshots from the MB-pol trajectory and Figures 2-4 with different $r_{\max }$ cutoffs.

\section{ACKNOWLEDGMENTS}

We are grateful to F. Paesani for providing structures from his PIMD MB-pol simulations of ambient water and to K.T. Wikfeldt for supplying the PIMD opt-PBE-vdW structures from ref. ${ }^{79}$. This work was supported by the Swedish Research Council (VR) through Grant number 2015-009559. Computational resources provided by the Swedish National Infrastructure for Computing (SNIC) at the HPC2N and PDC centers are gratefully acknowledged. NAB acknowledges support from the Leverhulme Trust through grant number RF-2014-231. 


\section{References}

1. L. G. M. Pettersson, R. H. Henchman and A. Nilsson, Chem. Rev. 116, 7459-7461 (2016).

2. M. F. Chaplin, Water Structure and Behavior. http://www.lsbu.ac.uk/water/index.html

3. A. Nilsson and L. G. M. Pettersson, Nature Commun. 6, 8998 (2015).

4. P. Gallo, K. Amann-Winkel, C. A. Angell, M. A. Anisimov, F. Caupin, C. Chakravarty, T. Loerting, A. Z. Panagiotopoulos, J. Russo, H. Tanaka, et al., Chem. Rev. 116, 7643-7500 (2016).

5. G. A. Cisneros, K. T. Wikfeldt, L. Ojamäe, J. Lu, Y. Xu, H. Torabifard, A. P. Bartók, G. Csányi, V. Molinero and F. Paesani, Chem. Rev. 116, 7501-7528 (2016).

6. P. Wernet, D. Nordlund, U. Bergmann, M. Cavalleri, M. Odelius, H. Ogasawara, L. Å. Näslund, T. K. Hirsch, L. Ojamäe, P. Glatzel, et al., Science 304, 995-999 (2004).

7. J. D. Smith, C. D. Cappa, K. R. Wilson, B. M. Messer, R. C. Cohen and R. J. Saykally, Science 306, 851 (2004).

8. A. Nilsson, P. Wernet, D. Nordlund, U. Bergmann, M. Cavalleri, M. Odelius, H. Ogasawara, L.-Å. Näslund, T. K. Hirsch, L. Ojamäe, et al., Science 308, 793a (2005).

9. C. Huang, K. T. Wikfeldt, T. Tokushima, D. Nordlund, Y. Harada, U. Bergmann, M. Niebuhr, T. M. Weiss, Y. Horikawa, M. Leetmaa, et al., Proc. Natl. Acad. Sci. (USA) 106, 15214-15218 (2009).

10. T. Tokushima, Y. Harada, Y. Horikawa, O. Takahashi, Y. Senba, H. Ohashi, L. G. M. Pettersson, A. Nilsson and S. Shin, J. El. Spec. Rel. Phen. 177, 192-205 (2010).

11. T. Tokushima, Y. Harada, O. Takahashi, Y. Senba, H. Ohashi, L. G. M. Pettersson, A. Nilsson and S. Shin, Chem. Phys. Lett. 460, 387-400 (2008).

12. C. Huang, T. M. Weiss, D. Nordlund, K. T. Wikfeldt, L. G. M. Pettersson and A. Nilsson, J. Chem. Phys. 133, 134504 (2010).

13. K. T. Wikfeldt, C. Huang, A. Nilsson and L. G. M. Pettersson, J. Chem. Phys. 134, 214506 (2011).

14. A. Nilsson and L. G. M. Pettersson, Chem. Phys. 389, 1-34 (2011).

15. A. Nilsson, C. Huang and L. G. M. Pettersson, J. Mol. Liq. 176, 2-16 (2012).

16. P. H. Poole, F. Sciortino, U. Essmann and H. E. Stanley, Nature 360, 324-328 (1992).

17. O. Mishima, J. Chem. Phys. 133, 144503 (2010).

18. O. Mishima and H. E. Stanley, Nature 396, 329-335 (1998).

19. O. Mishima and H. E. Stanley, Nature 392, 164-168 (1998).

20. J. C. Palmer, F. Martelli, Y. Liu, R. Car, A. Z. Panagiotopoulos and P. G. Debenedetti, Nature, 385-388 (2014).

21. R. S. Singh, J. W. Biddle, P. G. Debenedetti and M. A. Anisimov, J. Chem. Phys. 144, 144504 (2016).

22. F. Perakis, K. Amann-Winkel, F. Lehmkühler, M. Sprung, D. Pettersson, J. A. Sellberg, H. Pathak, A. Späh, F. Cavalca, D. Schlesinger, et al., Proc. Natl. Acad. Sci. (USA) 114, 8193-8198 (2017).

23. D. A. Fuentevilla and M. A. Anisimov, Phys. Rev. Lett. 97, 195702 (2006).

24. V. Holten and M. A. Anisimov, Sci. Reps. 2, 713 (2012).

25. C. A. Angell, Science 319, 582 (2008).

26. C. A. Angell, Nature Materials 13, 673-675 (2014).

27. R. J. Speedy and C. A. Angell, J. Chem. Phys. 65, $851-858$ (1976).

28. J. A. Sellberg, C. Huang, T. A. McQueen, N. D. Loh, H. Laksmono, D. Schlesinger, R. G. Sierra, D. Nordlund, C. Y. Hampton, D. Starodub, et al., Nature 510, 381-384 (2014).

29. G. N. I. Clark, G. Hura, J. Teixeira, A. K. Soper and T. Head-Gordon, Proc. Natl. Acad. Sci. (USA) 107, 14003-14007 (2010).

30. C. Huang, K. T. Wikfeldt, T. Tokushima, D. Nordlund, Y. Harada, U. Bergmann, M. Niebuhr, T. M. Weiss, Y. Horikawa, M. Leetmaa, et al., Proc. Natl. Acad. Sci. (USA) 107, E45 (2010).

31. A. K. Soper, Pure Appl. Chem. 82, 1855-1867 (2010).

32. A. K. Soper, J. Teixeira and T. Head-Gordon, Proc. National Acad. Sci. (USA) 107, E44 (2010).

33. S. D. Overduin and G. N. Patey, J. Phys. Chem. B 116, 12014-12020 (2012). 
34. J. L. F. Abascal and C. Vega, J. Chem. Phys. 123, 234505 (2005).

35. K.-H. Kim, A. Späh, H. Pathak, F. Perakis, D. Mariedahl, K. Amann-Winkel, J. A. Sellberg, J. H. Lee, S. Kim, J. Park, et al., Science 358, 1589-1593 (2017).

36. J. W. Biddle, R. S. Singh, E. M. Sparano, F. Ricci, M. A. González, C. Valeriani, J. L. F. Abascal, P. G. Debenedetti, M. A. Anisimov and F. Caupin, J. Chem. Phys. 146, 034502 (2017).

37. J. Russo and H. Tanaka, Nat. Commun. 5, 3556 (2014).

38. J. R. Scherer, M. K. Go and S. Kint, J. Phys. Chem. 78, 1304-1312 (1974).

39. Y. Maréchal, J. Mol. Struct. 1004, 146-155 (2011).

40. P. L. Geissler, J. Am. Chem. Soc. 127, 14930-14935 (2005).

41. J. D. Smith, C. D. Cappa, K. R. Wilson, R. C. Cohen, P. L. Geissler and R. J. Saykally, Proc. National Acad. Sci. (USA) 102, 14171 (2005).

42. S. K. Reddy, D. R. Moberg, S. C. Straight and F. Paesani, J. Chem. Phys. 147, 244504 (2017).

43. Y. Liu, J. C. Palmer, A. Z. Panagiotopoulos and P. G. Debenedetti, J. Chem. Phys. 137, 214505 (2012).

44. J. C. Palmer, R. Car and P. G. Debenedetti, Faraday Disc. 167, 77-94 (2013).

45. D. Chandler, Nature 531, E1-E2 (2016).

46. J. C. Palmer, F. Martelli, Y. Liu, R. Car, A. Z. Panagiotopoulos and P. G. Debenedetti, Nature 531, E2E3 (2016).

47. D. T. Limmer and D. Chandler, J. Chem. Phys. 135, 134503 (2011).

48. D. T. Limmer and D. Chandler, J. Chem. Phys. 138, 214504 (2013).

49. D. T. Limmer and D. Chandler, Mol. Phys. 113, 2799-2804 (2015).

50. F. Smallenburg and F. Scortino, Phys. Rev. Lett. 115, 015701 (2015).

51. T. A. Kesselring, G. Franzese, S. V. Buldyrev, H. J. Herrmann and H. E. Stanley, Sci. Reps. 2, 474 (2012).

52. T. A. Kesselring, E. Lascaris, G. Franzese and H. E. Stanley, J. Chem. Phys. 138, 244506 (2013).

53. F. H. Stillinger and A. Rahman, J. Chem. Phys. 60, 1545-1557 (1974).

54. J. C. Palmer, A. Haji-Akbari, R. S. Singh, F. Martelli, R. Car, A. Z. Panagiotopoulos and P. G. Debenedetti, J. Chem. Phys., in press (2018).

55. T. Head-Gordon and S. W. Rick, Phys. Chem. Chem. Phys. 9, 83-91 (2007).

56. S. Myneni, Y. Luo, L.-Å. Näslund, M. Cavalleri, L. Ojamäe, H. Ogasawara, A. Pelmenschikov, P. Wernet, P. Väterlein, C. Heske, et al., J. Phys.: Condens. Matter 14, L213-L219 (2002).

57. J. D. Smith, C. D. Cappa, B. M. Messer, W. S. Drisdell, R. C. Cohen and R. J. Saykally, J. Phys. Chem. B 110, 20038-20045 (2006).

58. R. Z. Khaliullin and T. D. Kühne, J. Am. Chem. Soc. 136, 3395-3399 (2014).

59. T. D. Kühne and R. Z. Khaliullin, Nat. Commun. 4, 1450 (2013).

60. T. Pylkkänen, V. M. Giordano, J.-C. Chervin, A. Sakko, M. Hakala, J. A. Soininen, K. Hämäläinen, G. Monaco and S. Huotari, J. Phys. Chem. B 114, 3804-3808 (2010).

61. T. Pylkkänen, A. Sakko, M. Hakala, K. Hämäläinen, G. Monaco and S. Huotari, J. Phys. Chem. B 115, 14544-14550 (2011).

62. G. N. I. Clark, C. D. Cappa, J. D. Smith, R. J. Saykally and T. Head-Gordon, Mol. Phys. 108, 1415-1433 (2010).

63. M. V. Fernandez-Serra and E. Artacho, Phys. Rev. Lett. 96, 016404 (2006).

64. C. J. Sahle, C. Sternemann, C. Schmidt, S. Lehtola, S. Jahn, L. Simonelli, S. Huotari, M. Hakala, T. Pylkkänen, A. Nyrow, et al., Proc. Natl. Acad. Sci. (USA) 110, 6301-6306 (2013).

65. E. T. J. Nibbering and T. Elsaesser, Chem. Rev. 104, 1887-1914 (2004).

66. A. Nilsson, D. Nordlund, I. Waluyo, N. Huang, H. Ogasawara, S. Kaya, U. Bergmann, L.-Å. Näslund, H. Öström, P. Wernet, et al., J. El. Spec. Rel. Phen. 177, 99-129 (2010).

67. M. Cavalleri, H. Ogasawara, L. G. M. Pettersson and A. Nilsson, Chem. Phys. Lett. 364, 363 (2002).

68. D. Nordlund, H. Ogasawara, K. J. Andersson, M. Tatarkhanov, M. Salmerón, L. G. M. Pettersson and A. Nilsson, Phys. Rev. B 80, 233404 (2009).

69. D. Nordlund, H. Ogasawara, P. Wernet, M. Nyberg, M. Odelius, L. G. M. Pettersson and A. Nilsson, Chem. Phys. Lett. 395, 161-165 (2004).

70. J. A. Sellberg, S. Kaya, V. H. Segtnan, C. Chen, T. Tyliszczak, H. Ogasawara, D. Nordlund, L. G. M. 
Pettersson and A. Nilsson, J. Chem. Phys. 141, 034507 (2014).

71. M. Leetmaa, M. P. Ljungberg, A. P. Lyubartsev, A. Nilsson and L. G. M. Pettersson, J. El. Spec. Rel. Phen. 177, 135-157 (2010).

72. M. Odelius, M. Cavalleri, A. Nilsson and L. G. M. Pettersson, Phys. Rev. B 73, 024205 (2006).

73. M. Iannuzzi, J. Chem. Phys. 128, 204506 (2008).

74. M. Iannuzzi and J. Hutter, Phys. Chem. Chem. Phys. 9, 1599 (2007).

75. W. Chen, X. Wu and R. Car, Phys. Rev. Lett. 105, 017802 (2010).

76. J. Vinson, J. J. Kas, J. J. Rehr, F. D. Vila and E. L. Shirley, Phys. Rev. B 85, 045101 (2012).

77. G. Brancato, N. Rega and V. Barone, Phys. Rev. Lett. 100, 107401 (2008).

78. D. Prendergast and G. Galli, Phys. Rev. Lett. 96, 215502 (2006).

79. T. Fransson, I. Zhovtobriukh, S. Coriani, K. T. Wikfeldt, P. Norman and L. G. M. Pettersson, Phys. Chem. Chem. Phys. 18, $566-583$ (2016).

80. T. Fransson, Y. Harada, N. Kosugi, N. A. Besley, B. Winter, J. Rehr, L. G. M. Pettersson and A. Nilsson, Chem. Rev. 116, 7551-7569 (2016).

81. L. Weinhardt, A. Benkert, F. Meyer, M. Blum, R. G. Wilks, W. Yang, M. Bär, F. Reinert and C. Heske, J. Chem. Phys. 136, 144311 (2012).

82. O. Fuchs, M. Zharnikov, L. Weinhardt, M. Blum, M. Weigand, Y. Zubavichus, M. Bär, F. Maier, J. D. Denlinger, C. Heske, et al., Phys. Rev. Lett. 100, 027801 (2008).

83. A. Pietzsch, F. Hennies, P. S. Miedema, B. Kennedy, J. Schlappa, T. Schmitt, V. N. Strocov and A. Föhlisch, Phys. Rev. Lett. 114, 088302 (2015).

84. K. M. Lange, R. Könecke, S. Ghadimi, R. Golnak, M. A. Soldatov, K. F. Hodeck, A. Soldatov and E. F. Aziz, Chem. Phys. 377, 1-5 (2010).

85. K. M. Lange, R. Könnecke, M. Soldatov, R. Golnak, J. E. Rubensson, A. Soldatov and E. F. Aziz, Angew. Chem. 123, 10809-10813 (2011).

86. K. M. Lange, M. Soldatov, R. Golnak, M. Gotz, N. Engel, R. Könnecke, J.-E. Rubensson and E. F. Aziz, Phys. Rev. B 85, 155104 (2012).

87. J. A. Sellberg, T. A. McQueen, H. Laksmono, S. Schreck, M. Beye, D. DePonte, B. O'Kennedy, D. Nordlund, R. G. Sierra, D. Schlesinger, et al., J. Chem. Phys. 142, 044505 (2015).

88. E. Gilberg, M. J. Hanus and B. Foltz, J. Chem. Phys. 76, 5093-5097 (1982).

89. O. Fuchs, M. Zharnikov, L. Weinhardt, M. Blum, M. Weigand, Y. Zubavichus, M. Bär, F. Maier, J. D. Denlinger, C. Heske, et al., Phys. Rev. Lett. 100, 249802 (2008).

90. K. Yamazoe, J. Miyawaki, H. Niwa, A. Nilsson and Y. Harada, Manuscript (2018).

91. M. Odelius, Phys. Rev. B 79, 144204 (2009).

92. M. Odelius, J. Phys. Chem. A 113, 8176-8181 (2009).

93. T. Tokushima, Y. Horikawa, H. Arai, Y. Harada, O. Takahashi, L. G. M. Pettersson, A. Nilsson and S. Shin, J. Chem. Phys. 136, 044517 (2012).

94. J. Forsberg, J. Gråsjö, B. Brena, J. Nordgren, L. C. Duda and J. E. Rubensson, Phys. Rev. B 79, 132203 (2009).

95. M. P. Ljungberg, L. G. M. Pettersson and A. Nilsson, J. Chem. Phys. 134, 044513 (2011).

96. A. Nilsson, T. Tokushima, Y. Horikawa, Y. Harada, M. P. Ljungberg, S. Shin and L. G. M. Pettersson, J. Electron. Spectrosc. Relat. Phenom. 188, 84-100 (2013).

97. L. G. M. Pettersson and A. Nilsson, J. Non-Crystalline Solids 407, 399-417 (2015).

98. N. A. Besley, Chem. Phys. Lett. 542, 42-46 (2012).

99. J. D. Wadey and N. A. Besley, J. Chem. Theory Comput. 10, 4557-4564 (2014).

100. B. Winter, E. F. Aziz, U. Hergenhahn, M. Faubel and I. V. Hertel, J. Chem. Phys. 126, 124504 (2007).

101. B. Winter, R. Weber, W. Widdra, M. Dittmar, M. Faubel and I. V. Hertel, J. Phys. Chem. A 108, 2625 (2004).

102. D. Nordlund, M. Odelius, H. Bluhm, H. Ogasawara, L. G. M. Pettersson and A. Nilsson, Chem. Phys. Lett. 460, 86 (2008).

103. M. Odelius, H. Ogasawara, D. Nordlund, O. Fuchs, L. Weinhardt, F. Maier, E. Umbach, C. Heske, Y. Zubavichus, M. Grunze, et al., Phys. Rev. Lett. 94, 227401 (2005). 
104. M. P. Ljungberg, A. Nilsson and L. G. M. Pettersson, Phys. Rev. B 82, 245115 (2010).

105. B. Brena, D. Nordlund, M. Odelius, H. Ogasawara, A. Nilsson and L. G. M. Pettersson, Phys. Rev. Lett. 93, 148302 (2004).

106. M. Neeb, J. E. Rubensson, M. Biermann and W. Eberhardt, J. El. Spec. Rel. Phen. 67, 261-274 (1994).

107. L. Weinhardt, M. Weigand, O. Fuchs, M. Bär, M. Blum, J. D. Denlinger, W. Yang, E. Umbach and C. Heske, Phys. Rev. B 84, 104202 (2011).

108. M. P. Ljungberg, I. Zhovtobriukh, O. Takahashi and L. G. M. Pettersson, J. Chem. Phys. 146, 134506 (2017).

109. O. Takahashi, M. P. Ljungberg and L. G. M. Pettersson, J. Phys. Chem. B 121, 11163-11168 (2017).

110. H. Shen, M. Chen, Z. Sun, L. Xu, E. Wang and X. Wu, Front. Phys. 13, 138204 (2018).

111. J. Niskanen, C. J. Sahle, K. Gilmore, F. Uhlig, J. Smiatek and A. Föhlisch, Phys. Rev. E 96, 013319 (2017).

112. Y. Harada, T. Tokushima, Y. Horikawa, O. Takahashi, H. Niwa, M. Kobayashi, M. Oshima, Y. Senba, H. Ohashi, K. T. Wikfeldt, et al., Phys. Rev. Lett. 111, 193001 (2013).

113. E. Shiratani and M. Sasai, J. Chem. Phys. 104, 7671-7680 (1996).

114. K. T. Wikfeldt, A. Nilsson and L. G. M. Pettersson, Phys. Chem. Chem. Phys. 13 19918-19924 (2011).

115. B. Santra, R. A. DiStasio Jr, F. Martelli and R. Car, Mol. Phys. 113, 2829-2841 (2015).

116. J. Klimeš, D. R. Bowler and A. Michaelides, J. Phys.: Cond. Mat. 22, 022201 (2010).

117. G. Kresse and J. Furthmüller, Comput. Mater. Sci. 6, 15-50 (1996).

118. G. Kresse and D. Joubert, Phys. Rev. B 59, 1758-1775 (1999).

119. V. Babin, G. R. Medders and F. Paesani, J. Phys. Chem. Lett. 3, 3765-3769 (2012).

120. G. R. Medders, V. Babin and F. Paesani, J. Chem. Theory Comput. 10, 2906-2910 (2014).

121. S. K. Reddy, S. C. Straight, P. Bajaj, C. H. Pham, M. Riera, D. R. Moberg, M. A. Morales, C. Knight, A. W. Gotz and F. Paesani, J. Chem. Phys. 145, 194504 (2016).

122. F. Paesani, Personal communication, (2016).

123. Y. H. Shao, Z. T. Gan, E. Epifanovsky, A. T. B. Gilbert, M. Wormit, J. Kussmann, A. W. Lange, A. Behn, J. Deng, X. T. Feng, et al., Mol. Phys. 113, 184-215 (2015).

124. M. E. Casida, in Recent Advances in Density Functional Methods, edited by D. P. Chong (World Scientific, Singapore, 1995), Vol. I.

125. S. Hirata and M. Head-Gordon, Chem. Phys. Lett. 314, 291-299 (1999).

126. A. T. B. Gilbert, N. A. Besley and P. M. W. Gill, J. Phys. Chem. A 112, 13161-13174 (2008).

127. O. Takahashi and L. G. M. Pettersson, J. Chem. Phys. 121, 10339-10345 (2004).

128. W. Kutzelnigg, U. Fleischer and M. Schindler, NMR-Basic Principles and Progress. (Springer Verlag, Heidelberg, 1990).

129. W. J. Hehre, R. Ditchfield and J. A. Pople, J. Chem. Phys. 56, 2257-2261 (1972).

130. P. Norman, D. M. Bishop, H. J. Aa. Jensen and J. Oddershede, J. Chem. Phys. 115, 10323-10334 (2001).

131. P. Norman, D. M. Bishop, H. J. Aa. Jensen and J. Oddershede, J. Chem. Phys. 123, 194103 (2005).

132. J. Kauczor and P. Norman, J. Chem. Theory Comput. 10, 2449-2455 (2014).

133. K. Aidas, C. Angeli, K. L. Bak, V. Bakken, R. Bast, L. Boman, O. Christiansen, R. Cimiraglia, S. Coriani, P. Dahle, et al., WIREs Comput. Mol. Sci. 4, 269-284 (2014).

134. T. Yanai, D. P. Tew and N. C. Handy, Chem. Phys. Lett. 393, 51-57 (2004).

135. U. Ekström, P. Norman, V. Carravetta and H. Ågren, Phys. Rev. Lett. 97, 143001 (2006).

136. U. Ekström and P. Norman, Phys. Rev. A 74, 042722 (2006).

137. A. Bergner, M. Dolg, W. Kuechle, H. Stoll and H. Preuß, Mol. Phys. 80, 1431-1441 (1993).

138. N. Godbout, D. R. Salahub, J. Andzelm and E. Wimmer, Can. J. Chem. 70, 560 (1992).

139. H. Ågren, V. Carravetta, O. Vahtras and L. G. M. Pettersson, Theoret. Chem. Acc. 97, 14 (1997).

140. S. Schreck, A. Pietzsch, K. Kunnus, B. Kennedy, W. Quevedo, P. S. Miedema, P. Wernet and A. Föhlisch, Struct. Dyn. 1, 054901 (2014).

141. L. G. M. Pettersson, T. Tokushima, Y. Harada, O. Takahashi, S. Shin and A. Nilsson, Phys. Rev. Lett. 100, 249801 (2008). 
142. F. K. Gel'mukhanov, L. N. Mazalov and A. V. Kondratenko, Chem. Phys. Lett. 46, 133 (1977).

143. L. Kong, X. Wu and R. Car, Phys. Rev. B 86, 134203 (2012).

144. K. Nishizawa, N. Kurahashi, K. Sekiguchi and T. Mizuno, Phys. Chem. Chem. Phys. 13, 413-417 (2011).

145. J. M. Guevara-Vela, E. Romero-Montalvo, V. A. M. Gómez, R. Chávez-Calvillo, M. García-Revilla, E. Francisco, Á. M. Pendás and T. Rocha-Rinza, Phys. Chem. Chem. Phys. 18, 19557-19566 (2016).

146. J. Stöhr, F. Sette and A. L. Johnson, Phys. Rev. Lett. 53, 1684 (1984).

147. L. B. Skinner, C. Huang, D. Schlesinger, L. G. M. Pettersson, A. Nilsson and C. J. Benmore, J. Chem. Phys. 138, 074506 (2013).

148. U. Bergmann, A. Di Cicco, P. Wernet, E. Principi, P. Glatzel and A. Nilsson, J. Chem. Phys. 127, 174504 (2007).

149. U. Bergmann, A. Di Cicco, P. Wernet, E. Principi, P. Glatzel and A. Nilsson, J. Chem. Phys. 128, 089902 (2008).

150. K. T. Wikfeldt, M. Leetmaa, A. Mace, A. Nilsson and L. G. M. Pettersson, J. Chem. Phys. 132, 104513 (2010).

151. M. Leetmaa, K. T. Wikfeldt and L. G. M. Pettersson, J. Phys.: Cond. Mat. 22, 135001 (2010).

152. M. Leetmaa, K. T. Wikfeldt, M. P. Ljungberg, M. Odelius, J. Swenson, A. Nilsson and L. G. M. Pettersson, J. Chem. Phys. 129, 084502 (2008).

153. Y. Harada, J. Miyawaki, H. Niwa, K. Yamazoe, L. G. M. Pettersson and A. Nilsson, J. Phys. Chem. Lett. 8, 5487-5491 (2017).

154. D. Nordlund, H. Ogasawara, H. Bluhm, O. Takahashi, M. Odelius, M. Nagasono, L. G. M. Pettersson and A. Nilsson, Phys. Rev. Lett. 99, 217406 (2007).

155. C. P. Lawrence and J. L. Skinner, J. Chem. Phys. 118, 264-272 (2003).

156. C. P. Lawrence and J. L. Skinner, Chem. Phys. Lett. 369, 472-477 (2003).

157. C. P. Lawrence and J. L. Skinner, J. Chem. Phys. 119, 1623-1633 (2003).

158. C. P. Lawrence and J. L. Skinner, J. Chem. Phys. 119, 3840-3848 (2003).

159. G. R. Medders and F. Paesani, J. Chem. Theory Comput. 11, 1145-1154 (2015).

160. G. R. Medders, A. W. Götz, M. A. Morales, P. Bajaj and F. Paesani, J. Chem. Phys. 143, 104102 (2015).

161. Q. Sun, Vib. Spectrosc. 62, 110-114 (2012).

162. Q. Sun, Chem. Phys. Lett. 568-569, 90-94 (2013). 


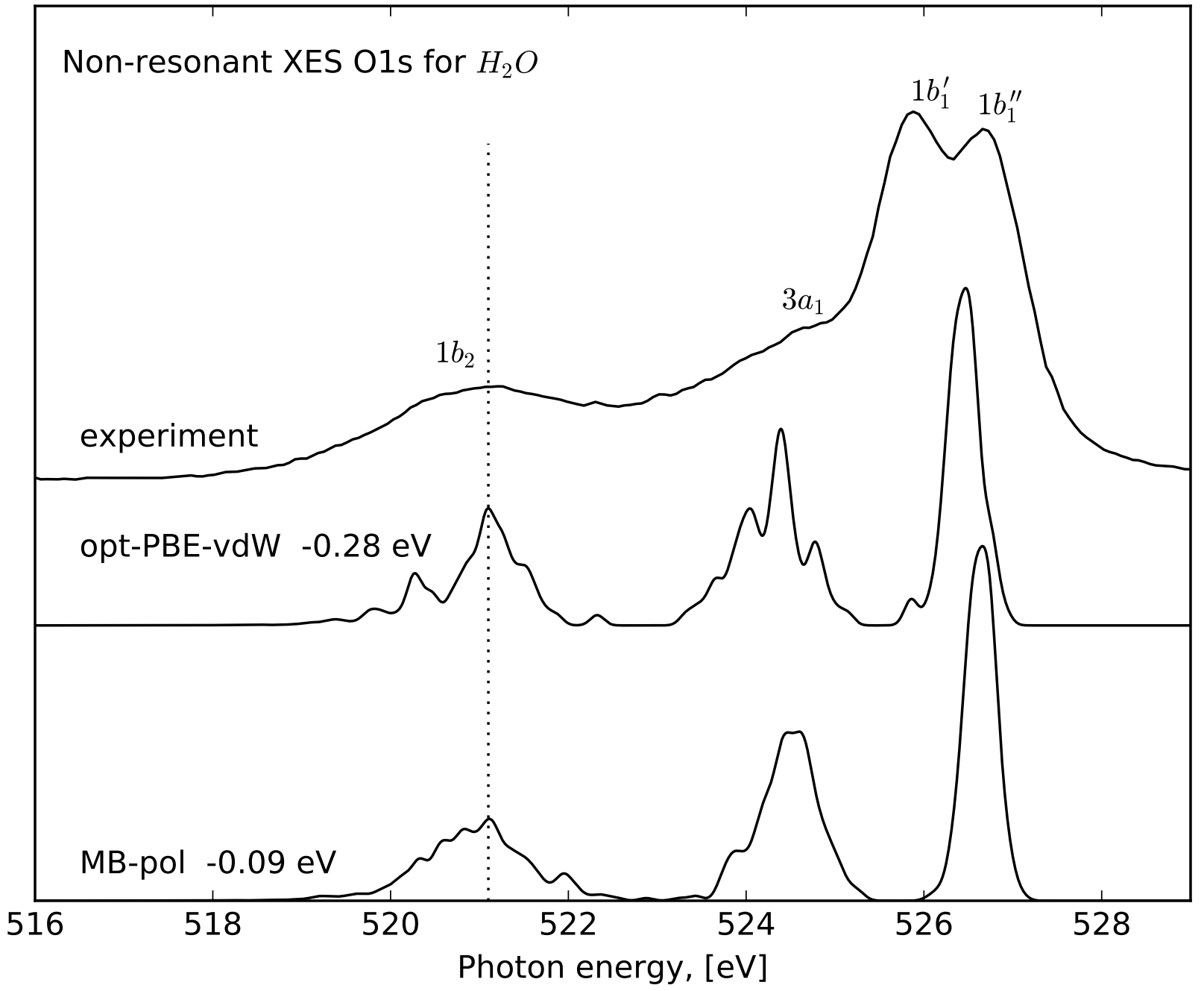



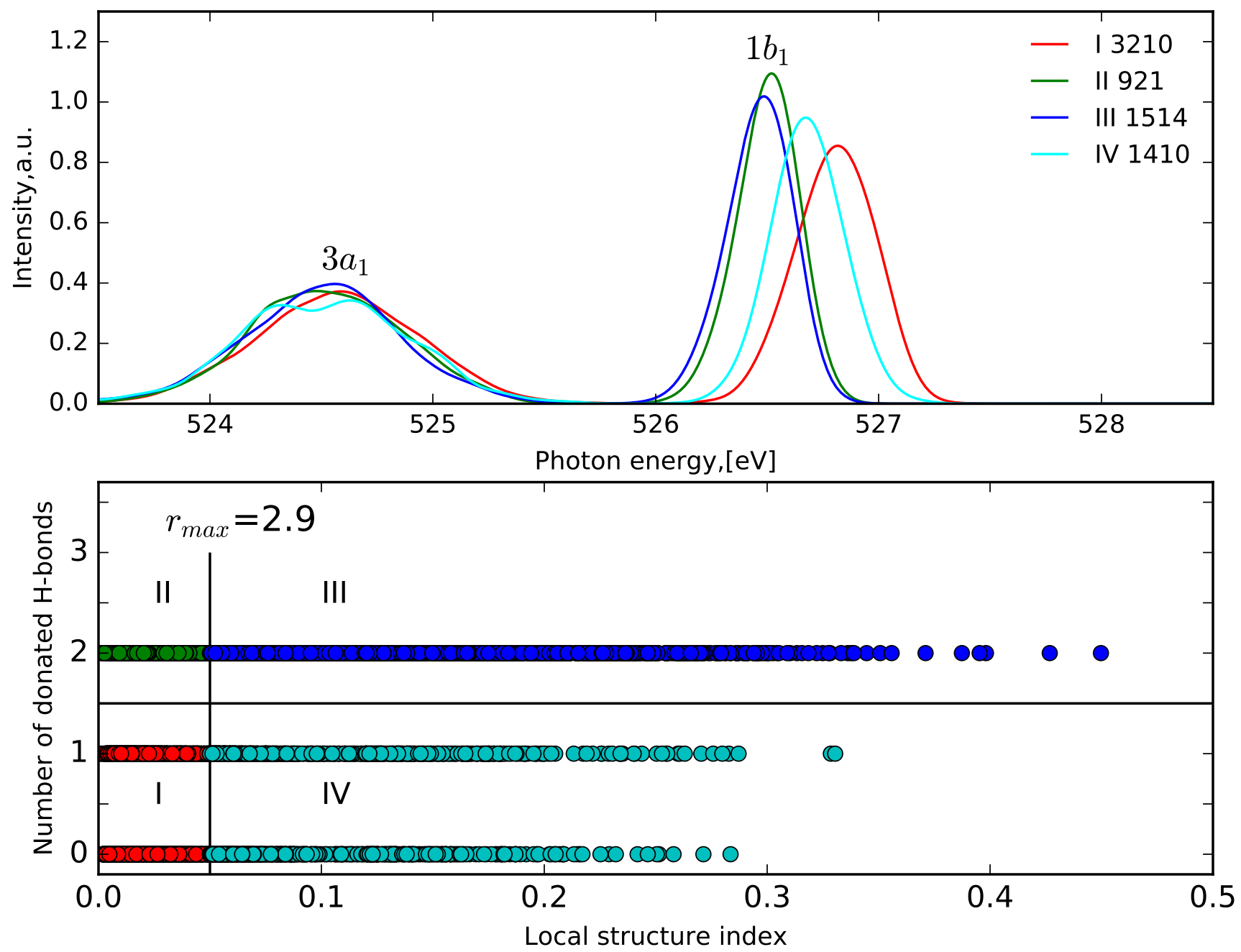

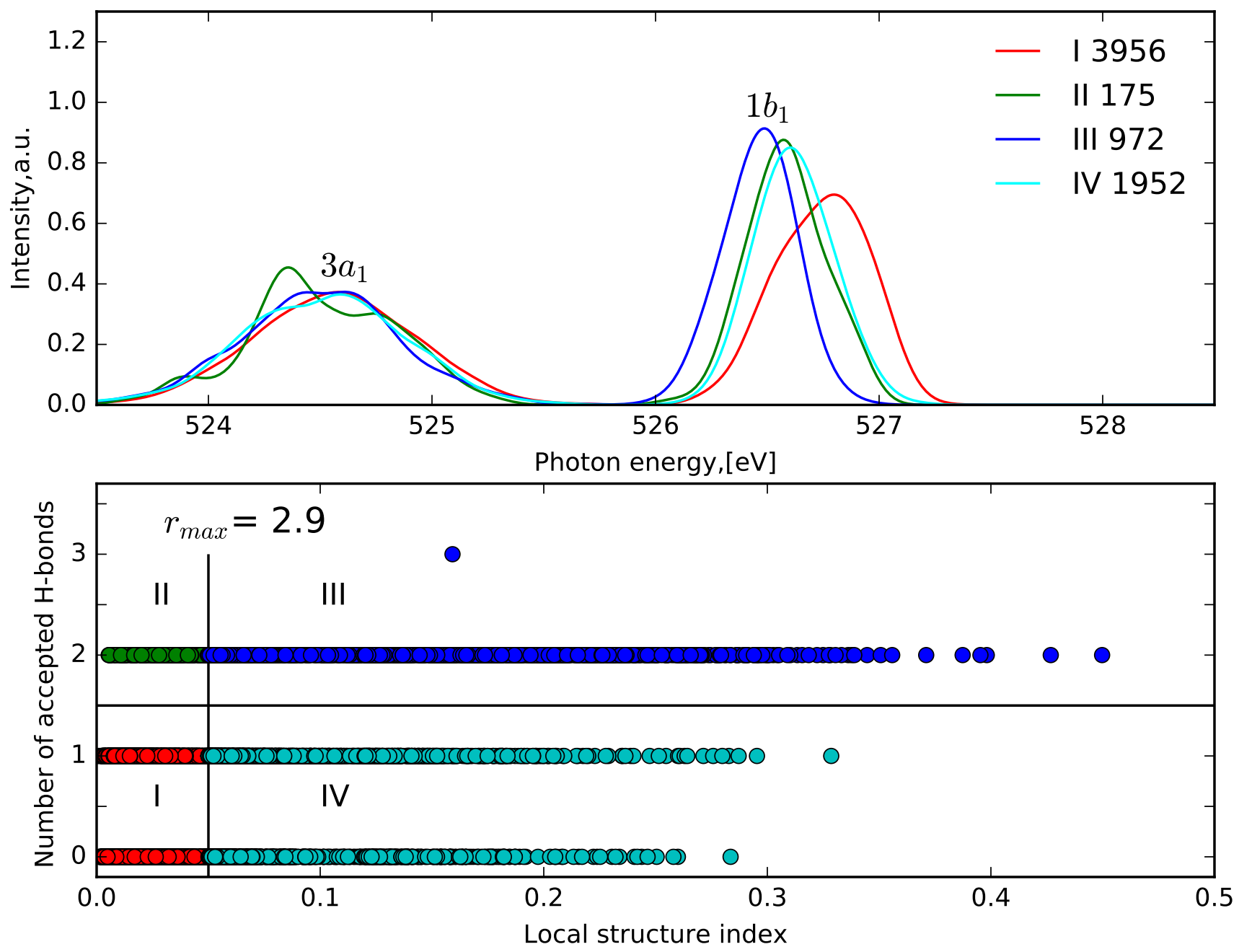

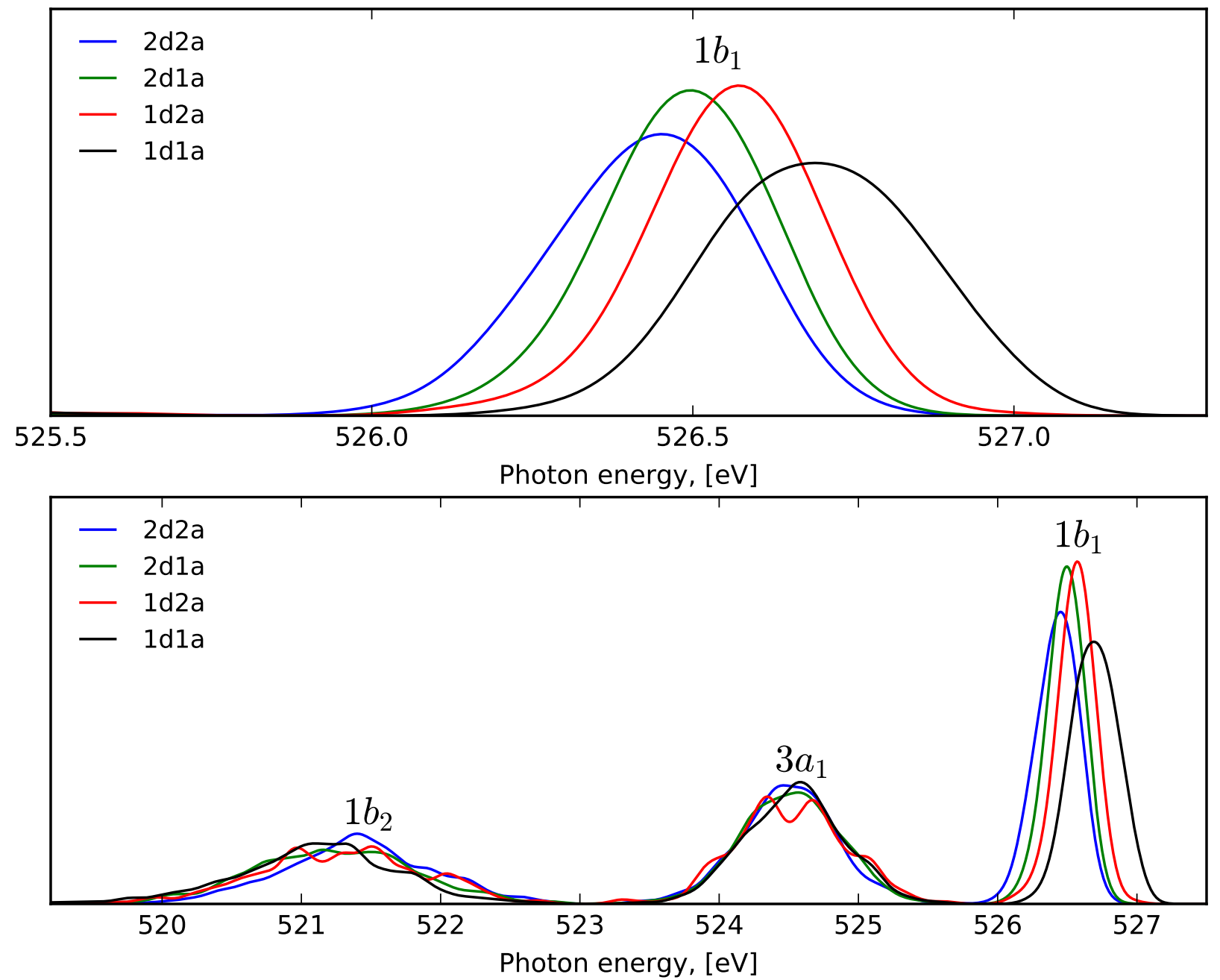


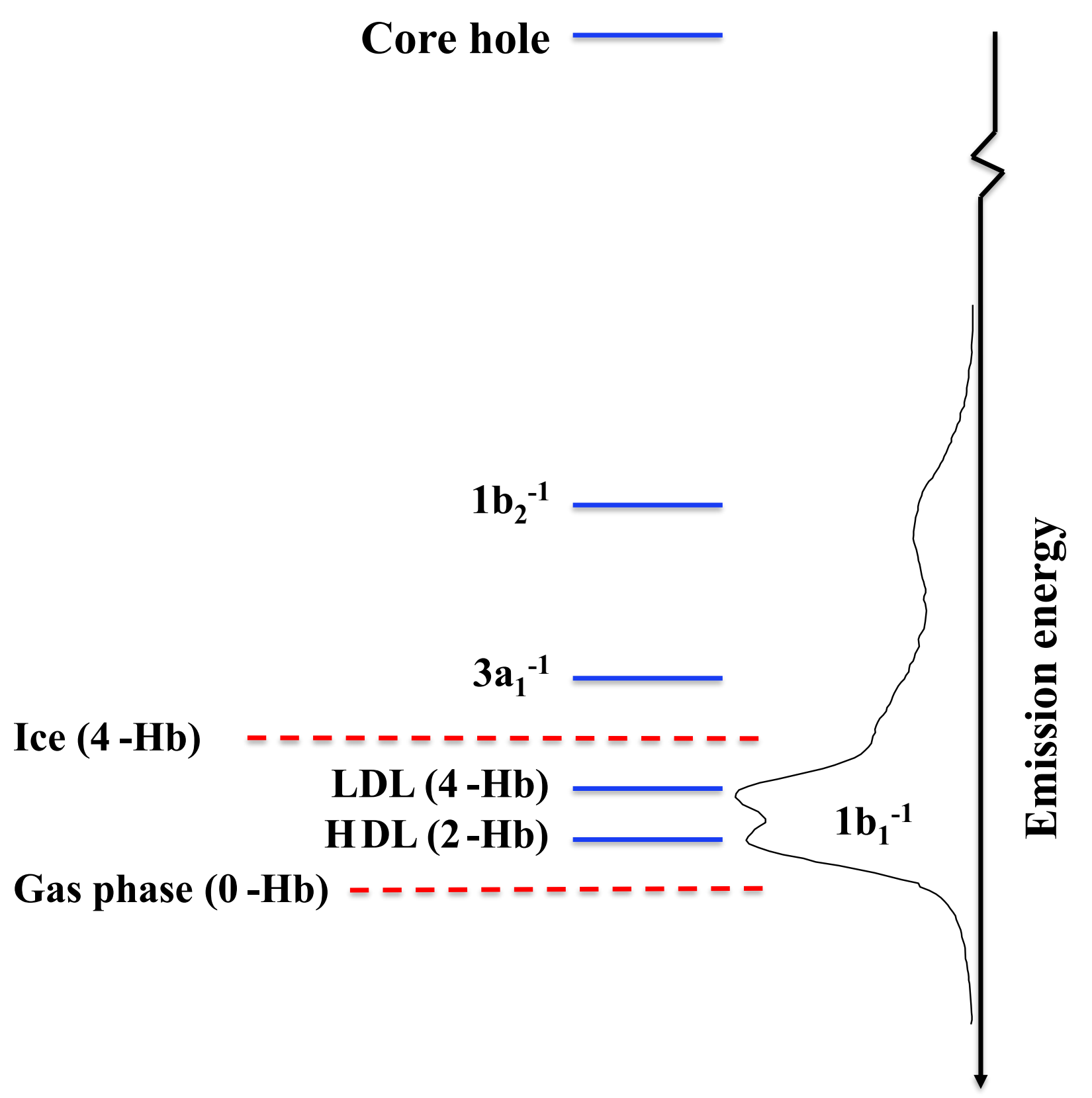



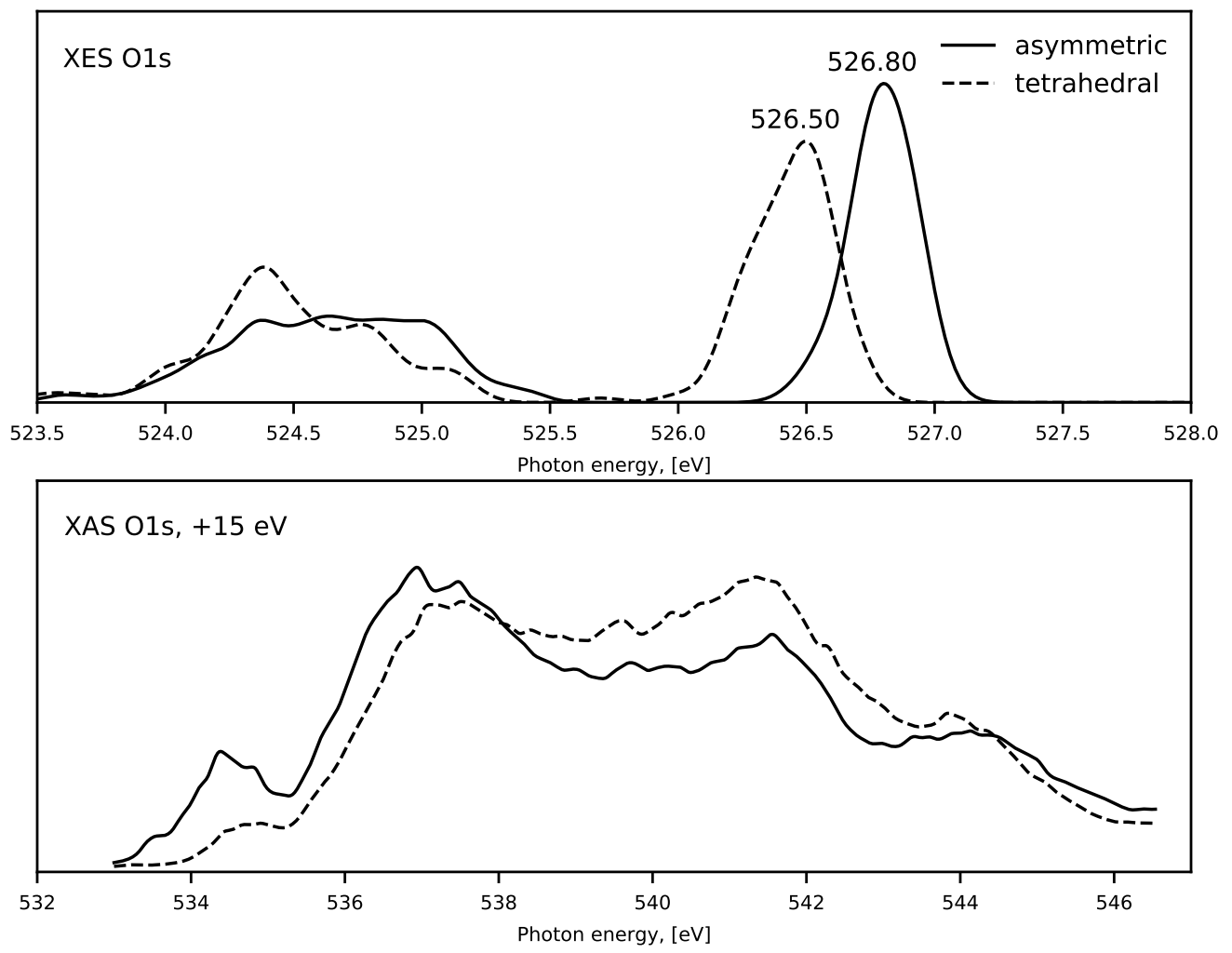

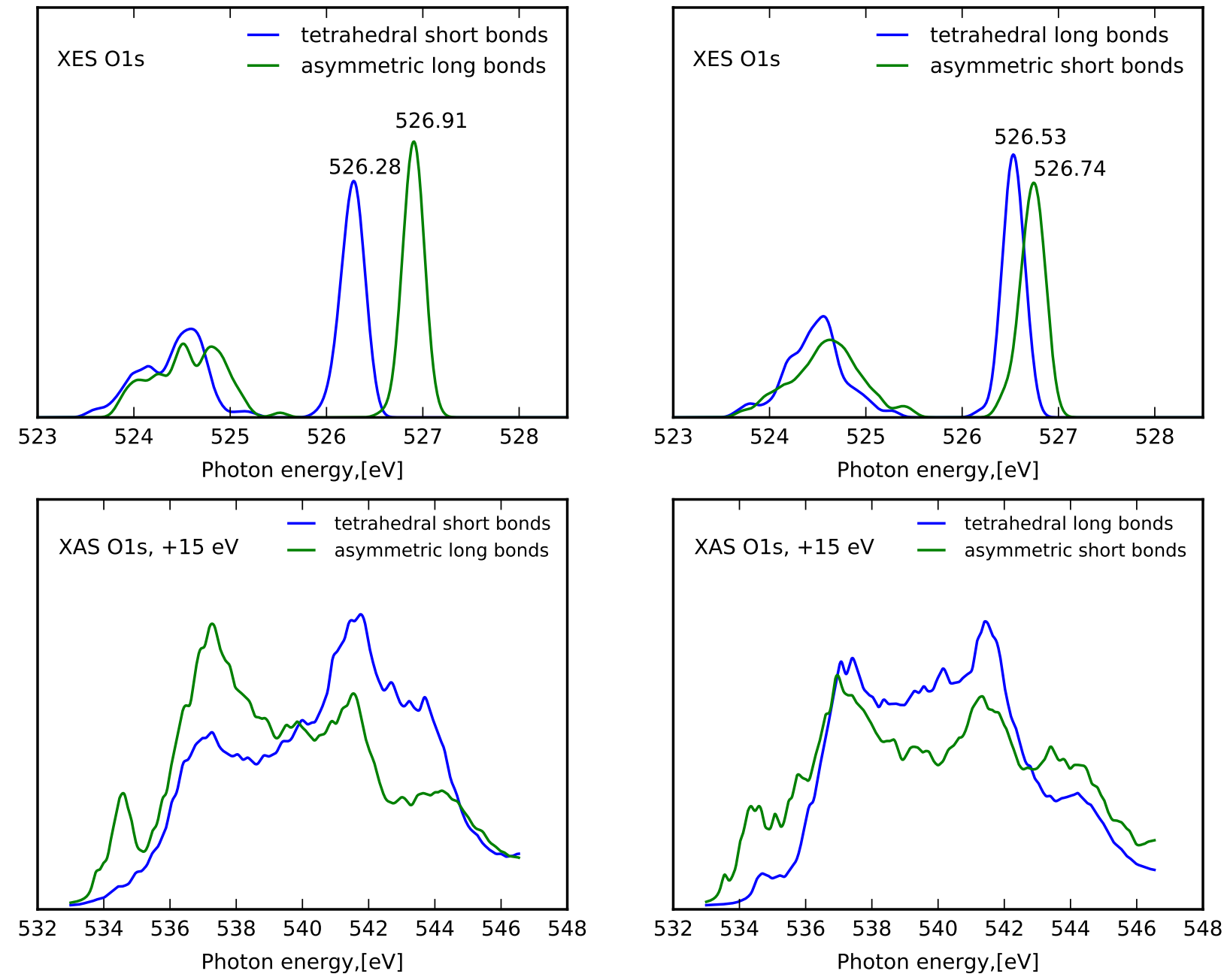


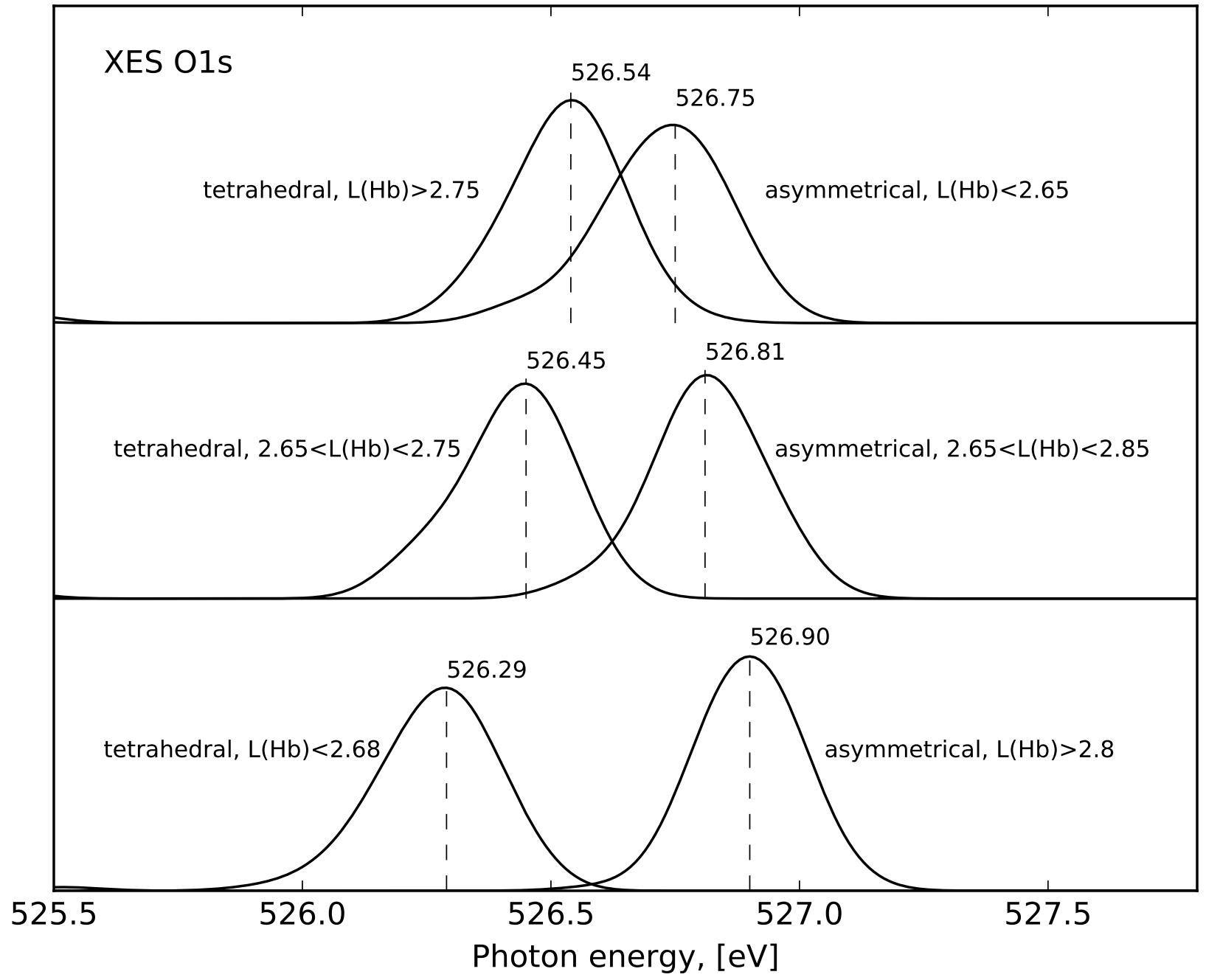



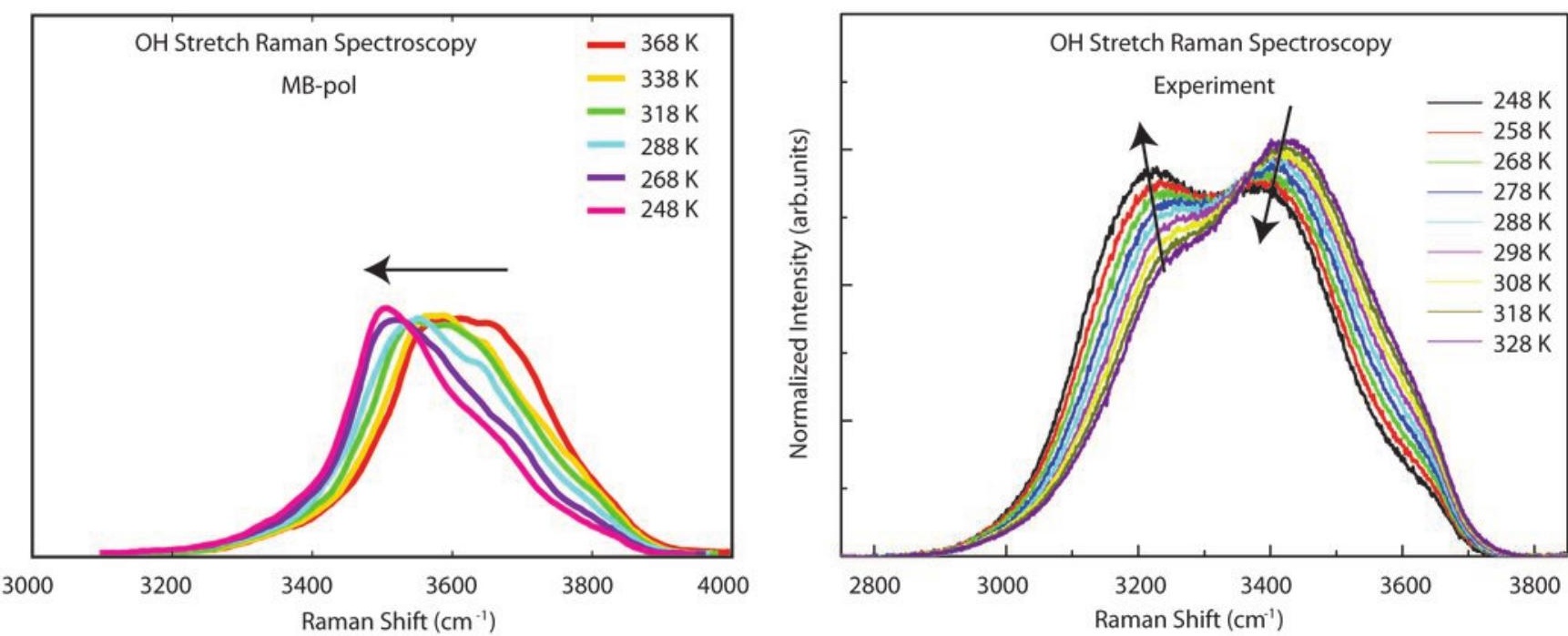